\title{
Operation Optimization of Integrated Energy Systems Based on Heat Storage Characteristics of Heating Network
}

\section{Ersheng PAN ( $\sim$ HBBDndyz@163.com )}

Stete Grid Beijing Economic Research Institute

Hui LI

State Grid Beijing Economic Research Institute

\section{Zhidong WANG}

State Grid Beijing Economic Research Institute

\section{Dong PENG}

State Grid Beijing Economic Research Institute

\section{Lang ZHAO}

State Grid Beijing Economic Research Institute

\section{Yongli WANG}

North China Electric Power University

\section{Feifei Huang}

North China Electric Power University https://orcid.org/0000-0002-4728-8641

\section{Yang MA}

North China Electric Power University

\section{Original article}

Keywords: integrated energy systems, dynamic heat energy transfer, virtual energy storage, operation optimization, economy

Posted Date: July 1st, 2020

DOl: https://doi.org/10.21203/rs.3.rs-37009/v1

License: (c) (i) This work is licensed under a Creative Commons Attribution 4.0 International License. Read Full License

Version of Record: A version of this preprint was published at Energy Science \& Engineering on November 22nd, 2020. See the published version at https://doi.org/10.1002/ese3.842. 


\title{
Operation Optimization of Integrated Energy Systems Based on Heat Storage
} Characteristics of Heating Network

Ersheng $\mathrm{PAN}^{1}$,Hui $\mathrm{LI}^{1}$, Zhidong WANG ${ }^{1}$, Dong $\mathrm{PENG}^{1}$, Lang ZHAO ${ }^{1}$, Yongli WANG ${ }^{2}$, Feifei

$$
\mathrm{HUANG}^{2} \text {, Yang MA }{ }^{2}
$$

(1. State Grid Beijing Economic Research Institute, Beijing 102209, China;

2. School of Economics and Management North China Electric Power University, Changping District, Beijing 102206 China)

\begin{abstract}
Background

With the rapid development of multi-energy technology and the wide application of electric-heat integrated energy system (IES), multi-energy network optimization has become an important research direction of multi-energy joint supply in recent years. Compared with electric energy, heat energy has the characteristics of delay in transmission time and fuzzy body temperature, which makes the heating system more flexible to participate in IES.
\end{abstract}

\section{Methods}

An operation optimization model for the electric-heat IES that takes into account the heat storage characteristics of the heating network is proposed to address the operation cost of the electric-heat IES. Firstly, the virtual energy storage characteristics of the heating network are proposed based on the characteristics of the transmission time delay of the heat system and the somatic blurring of user temperature. Then, the model of the dynamic transfer of energy in the heat system was built. Based on this, an operation optimization model of the electric-heat IES is established. And the Monte Carlo Simulation embedded Quantum Particle Swarm Optimization (MCS-QPSO) algorithm is proposed to solve the model.

\section{Results}

This paper takes a park in Northwest China as a simulation case to prove the effectiveness of the model. The analysis of the case shows that the model can optimize the output of energy supply equipment, so that the output equipment can maintain high efficiency. The mode changes the energy purchase strategy according to the price change of different energy. The results show that the proposed model can effectively utilize the virtual energy storage characteristics of the heating network to achieve energy transfer across time, thus reducing the operation cost of the IES.

\section{Conclusions}

In this paper, the operation optimization model of the electric heating IES is established based on the incubation period of the heating network and the virtual 
energy storage characteristics. The model can not only achieve the coordination and complementarity between the electric and thermal systems in time and space, but also improve the energy efficiency and reduce operation cost of the system.

Keywords: integrated energy systems; dynamic heat energy transfer; virtual energy storage; operation optimization; economy.

\section{Introduction}

Energy is the foundation of human survival and development and the lifeblood of the national economy. Under the premise of securing energy demand, how to reduce the operation cost of the system through rational dispatch of various energy sources has become the focus of world [1-2]. Among them, it is particularly important to break the current traditional model of energy systems being designed and operated separately, combine the various energy systems and increase the synergies among various energy systems [3]. With the deepening of the concept of energy internet, the IES as the physical carrier of energy internet has gradually attracted the attention of relevant scholars [4-5].

In order to enhance the energy supply capacity of the energy system and reduce the cost of energy supply. Some scholars have integrated the power system and the heat system, and established different forms of electro-heat coupling structure. Ref [6] proposed an electro-heat coupling model that takes into account the constraint of heat exchange link. Ref [7] analyzed various coupling units such as cogeneration, gas turbine, gas boiler, compressor, water pump, etc., and proposed an electro-heat coupling model of limited information interaction between networks. Ref [8] proposed an electro-heat coupling model of the overall energy flow based on the energy balance characteristics and the irreversibility of heat transport. Ref [9] proposed an electro-heat coupling model that takes into account the transmission losses of networks. The above mentioned study is aimed at connecting the electric and heat systems by establishing a medium (equipment) for the conversion of electric and heat energy.

In the field of operation optimization of IES with electro-heat coupling, Ref [10] proposed a control strategy for CCHP units based on the adoption of energy hubs. Ref [11] proposed an overall energy flow model based on the analysis of the energy and heat transport laws in IES. Ref [12] established energy network equations, generalized Kirchhoff's law from electric network theory to generalized Kirchhoff's law suitable for energy network modeling, and derived generalized concentrated parameter equivalent transport equations. Ref [13-14] constructed an electro-heat coupled network model and proposed an electro-heat mixed tide calculation model. The above 
Ref on the conversion relationship of energy flows is only applicable to static analysis, and further research is needed on the dynamic conversion of multi-energy flows.

Some scholars have also studied the thermal inertia of thermal system in the optimal operation of the electro-heat IES. Ref [15] studies the electric heat joint scheduling strategy of the distributed and coordinated control of the thermoelectric unit, the electric heating device and the energy storage device. Ref [16] proposed a multi-time scale energy coordination optimization method considering the variability of response speed in the energy flow change of the heating system. Ref [17] proposed a virtual energy storage system model based on the heat storage properties of the park heat energy. In the above study, the thermal inertia of thermal system is considered in the optimization of IES operation. However, the existing optimization scheduling model of electric-heat coupled IES often focuses on the use of thermal inertia service grid of thermal system to improve the permeability of renewable energy, without considering the incentive mechanism of virtual energy storage in the heat grid for demand response planning.

At present, although the independence of thermal power system integration has been considered in the existing research, the temperature dynamics and energy storage capacity of pipelines in regional heat supply network are seldom considered, so that the heat storage capacity of the regional heat supply network is not fully reflected. The temperature dynamics associated with the hot water flow and the heat storage effect of the district heat network have an important influence on the operation of the district heat network. In order to bridge this gap, this paper proposes a heating network model that takes into account the latency of the heat transfer process and the virtual energy storage characteristics of the primary heat network from the temperature dynamics of the district heat network, and establishes an electro-heat IES optimization scheduling model that takes into account the heat transfer dynamics characteristics. In this paper, the temperature of circulating water is adjusted without changing the number and flow of pipe network, the heat storage capacity of heat network is optimized, and the hydrological and thermal control decoupling of regional heat network system is realized. In addition, MCS-QPSO algorithm is proposed according to the characteristics of the model in this paper.

\section{Characterization and dynamic modeling of regional heat networks}

The virtual energy storage function of the heat network is complex, contradictory and dynamically changing. Modelling the dynamic processes of heat transport is key to reflect the latency and virtual energy storage characteristics of the heat network, which is helpful to the development of energy storage potential of the heat network 
and to participate in the optimal operation of electro-heat IES.

\subsection{Characterization of heat transfer}

The traditional energy system is limited to a single form of energy dispatch, such as electricity and heat, which does not take advantage of the complementary advantages of different energy sources. An IES can combine energy supply, energy conversion, energy storage, energy consumption and other physical processes into a unified energy supply system. The electric-heat IES combines the original independent electric and heat systems, which makes the whole system highly complementary. In the electric- heat IES, the power system mainly includes cogeneration units, photovoltaic panels, wind turbines, distribution grids, etc., who are responsible for the supply and transmission of electricity in the entire system. The heating system includes cogeneration, electric boiler, heat exchange station and heat network to meet the needs of users. The transmission of electric energy in the power system has the characteristics of real-time, fast and strong balance, and the storage of electric energy has the disadvantage of high cost and difficult storage. The heat transfer dynamics are described on a time scale of seconds, minutes or even hours, and are characterized by easy storage, difficult transmission, and transmission delays. And there is a certain ambiguity in the user's requirements for heat load. Therefore, the heat load has tunable properties over the electric load in terms of amplitude, a certain degree of panning in the timeline, etc. In addition, equipment such as cogeneration and electric boiler convert other energy sources into heat, coupling the electric and heat systems. As a result, integrated electric heating energy systems have strong complementary characteristics in energy production, energy supply, energy consumption, etc.

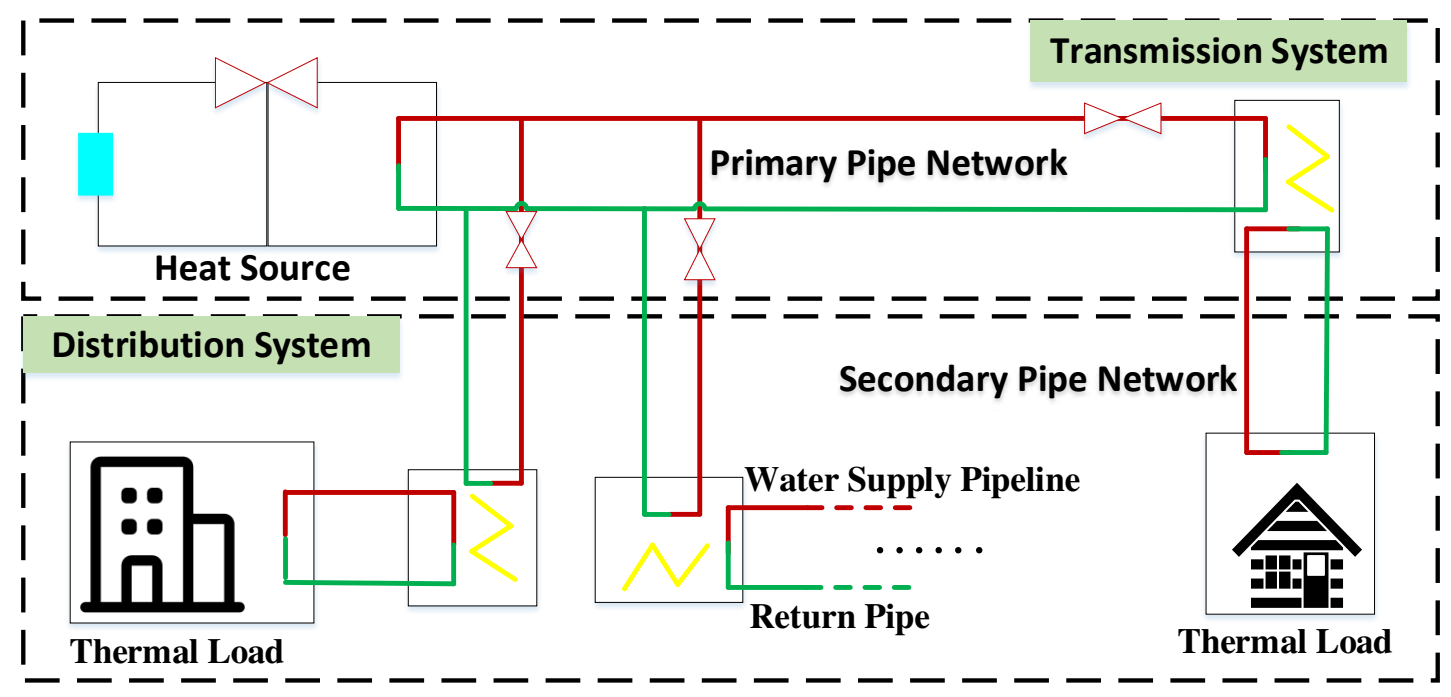

Fig.1. Architecture diagram of the heat system

A typical heat system consists of four parts: heat source, heat grid, heat exchange 
station and heat load [18]. Similar to electric systems, heat systems can be divided into transmission systems (primary pipeline network) and distribution systems (secondary pipeline network) [19]. The physical network of the primary and secondary pipelines is not connected, but heat exchange is carried out through heat exchange station. A heat exchange station is a heat load in the transmission system and a heat source in the distribution system. There are two main important features of the heat transfer process.

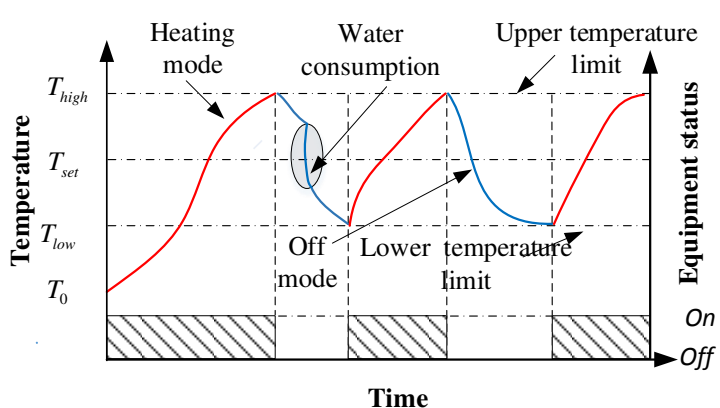

(a)

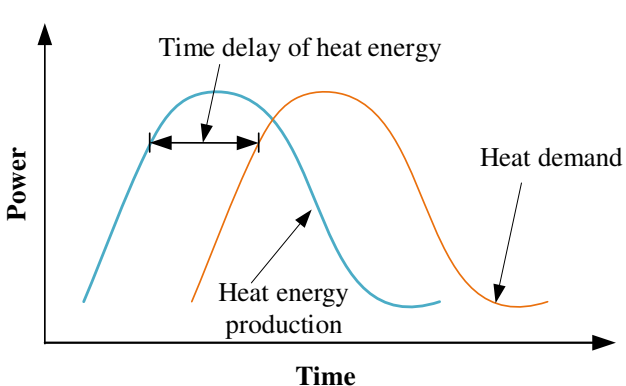

(b)

Fig. 2. Characterization of the heat system

i) Latency of transmission. The transfer of heat energy from the production facility to the user takes a certain amount of time, and changes in heat production are not immediately reflected on the user side.

ii) Ambiguity of heat sensation. A change in room temperature over a certain period of time will not affect the user experience and satisfaction.

Currently, the heat system calculates the temperature setting value for the secondary pipeline network water supply based on the outside temperature and the outside temperature compensation curve. Each heat station is reasonably set according to this value, and the electric valve on the primary pipe network is adjusted to achieve automatic flow or temperature regulation. This mode of operation achieves a balance between supply and demand for fixed heating delay times. Since the dynamic process of heat energy transfer has a large time scale, the heat of hot water entering and leaving the pipeline may not be equal at the same time, and the heat network can play the role of buffering energy and delaying the response [20], thus exhibiting the characteristics of virtual energy storage and recharge similar to energy storage systems. Considering the delayed and fuzzy of heat transfer, in the low price period, the heating facilities increase the power generation. The system controls the heat flow to each heat exchange station by regulating the electric valve, so that the heat is stored in the primary network. This mode of operation effectively takes advantage of the underestimation of electricity prices and the delay in the transmission of heat energy, enabling the transfer of heat energy across time. 


\subsection{Kinetic model of heat transfer in heating pipes}

The dynamic characteristics of heating pipes refers to the coupling relationship between the hot water inlet temperature and outlet temperature in the same pipe and its time, which is the key to describe the heat storage characteristics of the heating network. In a pipe, the change in water temperature at the inlet will slowly extend to the outlet, and the delay in temperature transfer will be essentially the same as the time the hot water flows through the pipe. In addition, due to the difference between the hot water temperature in the pipe and the ambient temperature, there will be heat loss during the flow resulting in a drop in water temperature. The cross-section of the heating pipe is shown in Fig.3, where $\Delta t$ is the length of the scheduling period.

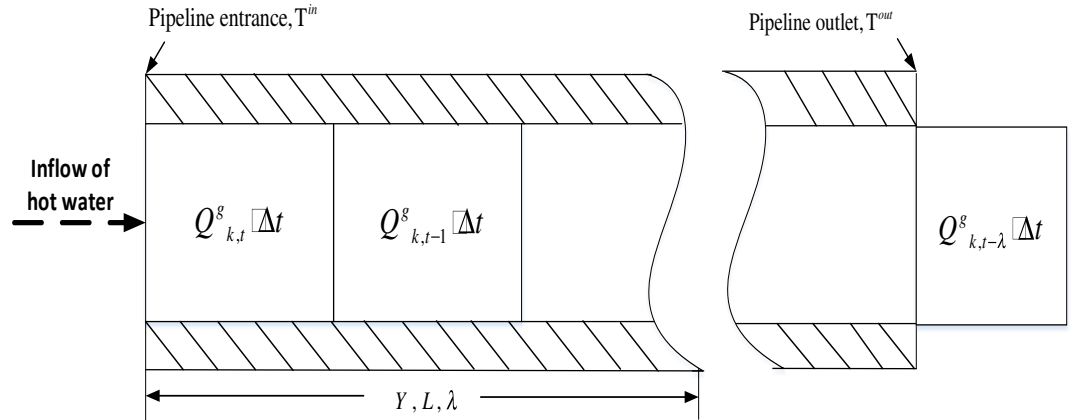

Fig.3. Cross-section of heating duct opening

(1)Assume that the time required for hot water to travel from inlet to outlet in pipe $k$ is $\lambda_{k, t}$ and there is no heat loss in between, then

$$
\begin{gathered}
T_{k, t}^{\text {out }}=T_{k, t^{-} \lambda_{k, t}}^{i n} \\
\lambda_{k, t}=\frac{Y_{k}}{Q_{k, t}^{g}}
\end{gathered}
$$

Equation (1) indicates that the discharge temperature $T_{k, t}^{\text {out }}$ of pipe $k$ at time $\mathrm{t}$ is equal to the inlet temperature $T_{k, t}^{i n} \lambda_{k, t}$ at time $t^{-} \lambda_{k, t}$ when heat loss of the pipe is not considered; the characteristic quantity $Y_{k}$ of pipe $k$ in equation (2) is determined by parameters such as pipe length and cross-sectional area.

(2) Calculate the effect of heat loss during transmission, as shown in equation (3)

$$
T_{k, t}^{\text {out }}=\left(T_{k, t}^{\text {out }} \cdot-T_{w, t}\right) \mathrm{r}^{\frac{-\alpha L_{k}}{c Q_{k, t}^{g}}}+T_{w, t}
$$

The upper equation can be approximated as:

$$
T_{k, t}^{\text {out }}=\left\{\begin{array}{cc}
\left(T_{k, t}^{\text {out }},-T_{w, t}\right)\left(1-\frac{\alpha L_{k}}{c Q_{k, t}^{g}}\right)+T_{w, t} & \frac{\alpha L_{k}}{c Q_{k, t}^{g}} \leq_{1} \\
T_{w, t} & \frac{\alpha_{L_{k}}}{c Q_{k, t}^{g}}>1
\end{array}\right.
$$

Where $T_{w, t}$ is the ambient temperature of the pipe; ${ }^{\alpha}$ is the heat conductivity 
of the pipe material; $L_{k}$ is the length of the pipe $k$.

During a scheduling period, if the heat source heat output is greater than (less than) the user's heat demand, the heat network virtual energy storage system plays an energy storage (or discharge) role, which is reflected by the return water temperature increases (or decreases) compared to the previous period. High return water temperature of the heat network will lead to an increase in heat network losses, while too low return water temperature will affect the heat transfer effect between the first and second heat network.

$$
T_{\min }^{\text {return }} \leq T^{\text {return }} \leq T_{\max }^{\text {return }}
$$

Where $T^{\text {return }}$ is the return water temperature of the first heat exchanger station, ${ }^{\circ} C ; T_{\max }^{\text {return }}$ is the upper design limit of pipeline return water temperature, corresponding to the upper limit of heat network energy storage; $T_{\min }^{\text {return }}$ is the lower design limit of pipeline return water temperature, corresponding to the upper limit of heat network energy discharge; the greater the difference between $T_{\max }^{\text {return }}$ and $T_{\mathrm{m} \text { in }}^{\text {return }}$, the stronger the heat network energy storage capacity.

\subsection{Nodal energy dynamic model}

Temperature regulation and flow regulation are two modes of regional heating network operation. In this paper, the temperature regulation method is used to control the temperature of the water supply and return, so as to control the energy in the heating network.

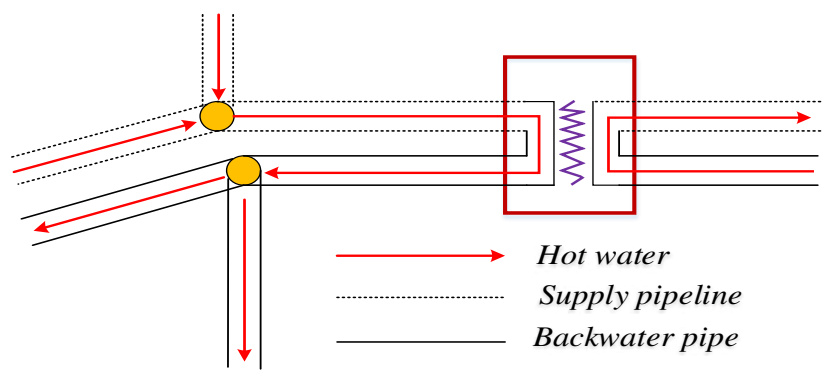

Fig.4. IES primary heat network structure diagram

(1) Nodal flow balance

That is, node mass flow continuity, so that the inflow of hot water into each node equals the outflow of mass flow, the specific expression is

$$
\sum_{n}^{N} d_{l, n}^{\text {in }}=\sum_{m}^{M} d_{l, m}^{\text {out }}
$$

Where $d_{l, n}^{\text {in }}$ represents the medium flow from branch $n$ to pipe section $l ; d_{l, m}^{\text {ou }}$ represents the medium flow from section $l$ to branch $m ; n$ and $m$ are the number of branches flowing in and out of the medium, respectively. 
(2) Pressure loss balance

The pressure loss in the pipeline is divided into the loss along the pipeline and the local loss. The loss along the pipeline is the pressure loss when the pipeline state is unchanged. Local loss is the pressure loss when the pipeline state changes.

$$
\begin{aligned}
& \Delta_{g_{f}}=\alpha \frac{l d^{2}}{4 r g} \\
& \Delta_{g_{j}}=\xi \frac{d^{2}}{2 g}
\end{aligned}
$$

Where ${ }^{\Delta_{g}}$ is the pressure loss along the pipeline section $l ;^{\Delta} g_{j}$ is the local pressure loss of pipe section $l ;{ }^{\alpha}$ and $\xi$ are the pressure loss coefficient of the resistance along the pipeline and the local resistance, respectively; $d$ is the flow through pipe $l ; r$ is the radius of pipe $l ; g$ is the acceleration of gravity.

In order to ensure the normal flow of medium in the pipeline, the lost pressure should be supplemented by the pump.

$$
\Delta_{g_{f}}+\Delta_{g_{j}}=g_{p}
$$

Where $g_{p}$ is the pressure provided by the pump.

(3) Heat energy balance

The energy contained in the flow of medium into and out of a certain pipe section is constant.

$$
\begin{aligned}
& \left\{\begin{array}{c}
Q_{l, n}^{\text {in }}=d_{l, n}^{\text {in }} c_{s} T_{l, n}^{\text {in }} / \rho \\
Q_{l, m}^{\text {out }}=d_{l, m}^{\text {out }} c_{s} T_{l, m}^{\text {out }} / \rho
\end{array}\right. \\
& \sum_{n}^{N} Q_{l, n}^{\text {in }}=\sum_{m}^{M} Q_{l, m}^{\text {out }}
\end{aligned}
$$

Where $Q_{l, n}^{i n}$ is the energy carried by the medium when it flows into the pipe section $l ; Q_{l, m}^{\text {out }}$ is the energy carried by the medium when it flows out of the pipe section $l ; c_{s}$ is the specific heat capacity of the medium, $\mathrm{kJ} /\left(\mathrm{kg} .{ }^{\circ} \mathrm{C}\right) ; \quad \rho$ is the density of the medium.

(4) Heat exchange station model

For the first station of heat exchange in the primary heat network, the load and loss can be bound together to become the calculated load of the first station of heat exchange, replacing the user load and the heat loss of the heat exchanger and the secondary heat network in the model, following the concept of calculated load in power system analysis. The first heat exchange station model in this paper is: 


$$
Q_{\lambda, \mathrm{i}}=c_{s} w_{\lambda, \mathrm{i}}\left(T_{\lambda, \mathrm{i}}^{\text {supply }}-T_{\lambda, \mathrm{i}}^{\text {return }}\right)
$$

Where $Q_{\lambda, \mathrm{j}}$ is the period load at the first station of heat exchange, $\mathrm{kJ} ; w_{\lambda, \mathrm{i}}$ is the mass of hot water flowing through the first station of heat exchange at load $i$ in time $\lambda, \mathrm{kg} ; T_{\lambda, \mathrm{i}}^{\text {supply }}{ }_{\lambda}$ and $T_{\lambda, \mathrm{i}}^{\text {return }}$ are the temperature of water supply and return water at the first station of heat exchange at period $\lambda$ respectively, ${ }^{\circ} C$.

The heat exchange station acts as a connection between the primary and secondary heat networks by regulating the flow in the pipes of the secondary heat network to specifically meet the user's heat load requirements. The relationship between heat exchange station heat exchange and heat load is:

$$
Q_{\lambda, \mathrm{i}}^{\text {load }} \Delta_{t}=Q_{\lambda, \mathrm{i}}
$$

Where $Q_{\lambda, \mathrm{i}}^{\text {load }}$ is the calculated heat load of the heat exchanger station at load $\mathrm{i}$ in time $\lambda, \mathrm{kW}$.

\section{Optimal Scheduling Model}

\subsection{Objective function}

The main goal of electro-heat IES optimization scheduling that takes into account the dynamic characteristics of heat energy transport is to minimize the operation cost of electro-heat IES while meeting the electric and heat load demands of the users. Operation cost include the electricity purchase cost for the upstream grid and the gas purchase cost and maintenance cost. The objective function of the electro-heat IES optimization scheduling model considering the dynamic properties of heat energy transport as follow.

$$
\operatorname{minC}=\mathrm{C}_{\text {grid }}+\mathrm{C}_{\text {gas }}+\mathrm{C}_{m c}
$$

Where $\mathrm{C}$ is the operation cost, Yuan; $\mathrm{C}_{\text {grid }}$ is the power purchase cost, Yuan; $\mathrm{C}_{g a s}$ is the gas purchase cost, Yuan; $C_{m c}$ is the operation and maintenance cost, Yuan.

$$
\begin{gathered}
\mathrm{C}_{\text {grid }}=\sum_{\lambda} F_{\lambda}{ }^{g r i d}\left(\mathrm{P}_{\lambda}{ }^{g r i d}+\mathrm{P}_{\lambda}{ }^{E B}\right) \Delta_{t} \\
\mathrm{C}_{\text {gas }}=\sum_{\lambda} F_{g a s} V_{\lambda}{ }^{g a s} \\
C_{m c}=\sum_{i=1}^{n} x^{i} w^{i}
\end{gathered}
$$

Where $F_{\lambda}{ }^{\text {grid }}$ is the electricity price in time ${ }^{\lambda}$, Yuan $/ \mathrm{kWh} ; \mathrm{P}_{\lambda}{ }^{\text {grid }}$ is the power purchased by the system from the upstream grid to meet the load in time ${ }^{\lambda}, \mathrm{kW} ; \mathrm{P}_{\lambda}{ }^{{ }^{B}}$ is the power of EB in time $\lambda ; F_{g a s}$ is the gas price, Yuan $/ \mathrm{m}^{3} ; V_{\lambda}{ }^{g a s}$ is the volume of 
gas purchased by the system from the upstream gas grid in time $\lambda, \mathrm{m}^{3} ; w^{i}$ is the maintenance cost of a single unit, Yuan.

\subsection{Constrains}

(1) Electric power balance constraints

$$
\begin{gathered}
P_{\lambda}^{C H P}+P_{\lambda}^{\text {grid }}-P_{\lambda}^{\text {lost }}=\sum_{i} P_{\lambda, i}^{\text {load }} \\
P_{\lambda}^{\text {lost }}=3 F I_{\max }^{2} R 10^{-3}
\end{gathered}
$$

Where $P_{\lambda}^{C H P}$ is the CHP electric power in the $\lambda$ period, $\mathrm{kW} ; \sum_{i} P_{\lambda, i}^{\text {load }}$ is the electric load of load node $\mathrm{i}$ in the $\lambda$ period, $\mathrm{kW} ; P_{\lambda}^{\text {lost }}$ is the electric system network loss in the $\lambda$ period, $\mathrm{kWh} ; F$ is the loss factor, equal to the ratio of the square of the root mean squared current to the square of the maximum current; $R$ is the line resistance, $\Omega ; I_{\max }$ is the maximum load current in the $\lambda$ period, A.

(2) Output and state change constraints of the device.

$$
\begin{gathered}
Q_{\text {min }}^{C H P} \leq Q_{\lambda}^{C H P} \leq Q_{\text {max }}^{C H P} \\
Q_{\text {min }}^{E B} \leq Q_{\lambda}^{E B} \leq Q_{\text {max }}^{E B} \\
-D_{\text {max }}^{C H P} \Delta_{\mathrm{t}} \leq P_{\lambda+1}^{C H P}-P_{\lambda}^{C H P} \leq U_{\text {max }}^{C H P}
\end{gathered}
$$

Where the subscripts min and max indicate the upper and lower limits of force output, respectively; $U_{\max }^{{ }^{C H P}}$ and $D_{\max }^{C H^{P}}$ are the limits of CHP's upward and downward climbing rates, respectively.

(3) Electric network transmission constraints

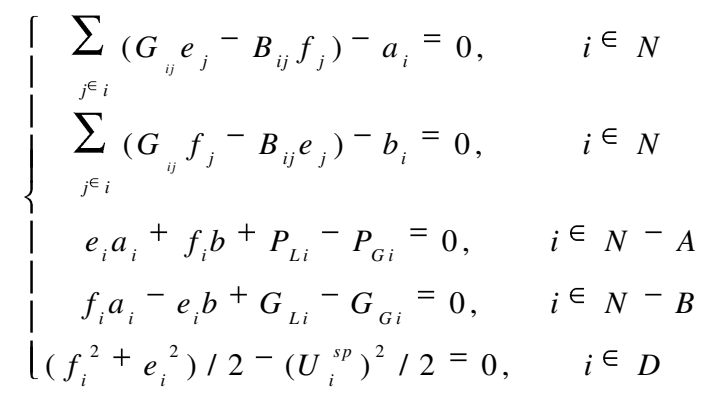

$$
\begin{aligned}
& \left\{\begin{array}{cc}
P_{G i}^{\min } \leq e_{i} a_{i}+f_{i} b+P_{L i} \leq P_{G i}^{\max }, & i \in A \\
G_{G i}^{\min } \leq f_{i} a_{i}-e_{i} b+G_{L i} \leq G_{G i}^{\max }, & { }_{i} \in B \\
\left(U_{i}^{\min }\right)^{2} / 2 \leq\left(f_{i}{ }^{2}+e_{i}{ }^{2}\right) / 2 \leq\left(U_{i}^{\max }\right)^{2} / 2 & i_{i} \in{ }_{N}-D
\end{array}\right.
\end{aligned}
$$

In Equations (29-30), $P_{G i}, G_{G i}, P_{L i}$ and $G_{L i}$ are the active and reactive power injected into node $\mathrm{i}$ by the generator and the active and reactive power absorbed by the load from node $\mathrm{i}$, respectively; ${ }_{U_{i}^{\mathrm{g}}}^{\mathrm{g}}$ is the node voltage; ${ }_{I_{i}^{\mathrm{g}}}^{\mathrm{g}}$ is node current; A is the 
set of nodes $(\mathrm{m} 1, \mathrm{~m} 1 \geq 1)$ that inject active power relaxation; $B$ is the set of nodes $(\mathrm{m} 2$, $\mathrm{m} 2 \geq 1$ ) that inject reactive power relaxation; D is the set of nodes ( $\mathrm{m} 3$ ) specified by the voltage magnitude.

(4) Heat transfer first station constraints

$$
\begin{array}{r}
Q_{\lambda}^{C H P}+Q_{\lambda}^{E B}=c_{r} w_{\lambda, \mathrm{m}}\left(T_{\lambda, \mathrm{i}}^{\text {supply }}-T_{\lambda, \mathrm{i}}^{\text {return }}\right) \\
T_{\text {min }}^{\text {supply }} \leq T^{\text {supply }} \leq T_{\text {max }}^{\text {supply }}
\end{array}
$$

Where $Q_{\lambda}^{C H P}$ and $Q_{\lambda}^{E B}$ are the heat power of $\mathrm{CHP}$ and $\mathrm{EB}$ at time $\lambda$ respectively; $w_{\lambda, \mathrm{m}}$ is the mass of hot water flowing through the first heat exchanger station at time $\lambda$.

(5) In order to facilitate the analysis of the virtual energy storage regulation potential of the heat network in the electro-heat IES optimization scheduling, the virtual energy storage, charge and discharge power of the heat network is defined as

$$
G^{-} Q^{-} Q^{\prime}
$$

Where $G$ is the virtual storage and discharge power of the heat network, "storage" is positive, "discharge" is negative; $Q$ is the heat output of the system when considering the dynamic characteristics of heat energy transfer; $Q$ is the heat output of the system without considering the dynamic characteristics of heat energy transfer, the heat output of the system under the scheduling scheme should always meet the demand for heat supply.

Other constraints on heat systems are described in equations (6)-(9).

\section{Solving algorithm}

\subsection{Variable identification}

The type and quantity of variables will affect the accuracy and time of model solution. Therefore, the variable identification is a key step to realize the accurate solution of the operation optimization model of the IES.

Tab.1 Variable identification in the model

\begin{tabular}{llc}
\hline \multirow{2}{*}{ Objective function } & \multicolumn{2}{c}{$\operatorname{minC}=\mathrm{C}_{\text {grid }}+\mathrm{C}_{\text {gas }}+C_{m c}$} \\
\cline { 2 - 3 } Variables & Integer & \multicolumn{1}{c}{ Mathematical characteristics } \\
\cline { 2 - 3 } & Non-integer & $F_{\lambda}{ }^{\text {grid }}, \mathrm{P}_{\lambda}{ }^{g \text { rid }}, \mathrm{P}_{\lambda}{ }^{E B}, F_{\text {gas }}, V_{\lambda}{ }^{g a s}, w^{i}$ \\
\hline
\end{tabular}

As shown in Tab. 1, the structure of optimization model is very complex, including many non-integer variables and integer variables. In addition, the coupling between electric network and thermal network is nonlinear. Therefore, the operation optimization of comprehensive energy system is a complex nonlinear optimization problem.

\subsection{Solution algorithm}


In order to solve the nonlinear optimization problem in the integrated energy system, this paper proposes a Monte Carlo Simulation embedded Quantum Particle Swarm Optimization (MCS-QPSO) algorithm to solve the problem. MCS-QPSO algorithm uses qubits to encode the current position of particles, and uses quantum rotating gate to search the optimal position of particles [22]. MCS-QPSO algorithm improves its global searching ability by implementing mutation operation through quantum non-gate. MCS-QPSO algorithm solves the uncertain problems such as wind speed, light and load growth during the operation optimization of IES by Monte Carlo simulation. The main steps of MCS-QPSO algorithm are as follows.

(1) Solution space transformation

$$
\left\{\begin{array}{l}
x_{m n}=\delta_{m n}\left(u_{m n}-l_{m n}\right)+u_{m n} \\
y_{m n}=\varsigma_{m n}\left(u_{m n}-l_{m n}\right)+u_{m n}
\end{array}\right.
$$

Where $\delta_{m n}$ and $\varsigma_{m n}$ represents the probability amplitude of the $n$ dimension of particle $m ; x_{m n}$ and $y_{m n}$ is the solution space variable; $u_{m n}$ and $l_{m n}$ represent the upper and lower limits of the search range of particle $m$.

(2) Solution space phase shift

$$
\begin{aligned}
& \left.\Delta \underset{m n}{\Delta \vartheta^{\left(j^{+1}\right)}}=\beta \Delta \vartheta_{m n}^{(j)}+s_{1} r_{1}^{(j)} \underset{p m n}{\vartheta_{p(j)}^{(j)}}-\vartheta_{m n}^{(j)}\right)+s_{2} r_{2}^{(j)}\left(\underset{g n}{\vartheta^{(j)}}-\vartheta_{m n}^{(j)}\right) \\
& {\left[\begin{array}{l}
\cos \vartheta_{m n}^{\left(j^{+} 1\right)} \\
\sin \vartheta_{m n}^{\left(j^{+}\right)}
\end{array}\right]=\left[\begin{array}{c}
\cos \vartheta_{m n}^{(j)} \\
\sin \vartheta_{m n}^{(j)}
\end{array}\right] \cdot\left[\begin{array}{cc}
\cos \Delta \vartheta_{m n}^{\left(j^{+} 1\right)} & -\sin \Delta \vartheta_{m n}^{\left(j^{+}\right)} \\
\sin \Delta \vartheta_{m n}^{\left(j^{+} 1\right)} & \cos \Delta \vartheta_{m n}^{\left(j^{+} 1\right)}
\end{array}\right]}
\end{aligned}
$$

Where $\underset{m n}{\vartheta^{(j)}}$ represents the phase in the $n$ dimension of particle $m$ in the $j$ iteration. $\vartheta_{p m n}^{(j)}$ and $\vartheta_{g n}^{(j)}$ represent the historical optimal phase of the $n$ dimension and the global optimal phase of the nth dimension of particle $m$ in the $j$ iteration, respectively; $\cos \vartheta_{m n}^{(j)}$ and $\sin \vartheta_{m n}^{(j)}$ represent the probability amplitude of the $n$ dimension of particle $m$ in the $j$ iteration; $s_{1}$ and $s_{2}$ are acceleration coefficient; $\beta$ is the inertia factor; $r_{1}$ and $r_{2}$ are random number with ranges of $[0,1]$.

(3) Mutation operation

$$
\left[\begin{array}{ll}
0 & 1 \\
1 & 0
\end{array}\right]\left[\begin{array}{l}
\cos { }_{m n} \\
\sin \vartheta_{m n}
\end{array}\right]=\left[\begin{array}{l}
\cos { }_{m n} \\
\sin \vartheta_{m n}
\end{array}\right]=\left[\begin{array}{l}
\cos \left(0.5 \pi-\vartheta_{m n}\right) \\
\sin \left(0.5 \pi-\vartheta_{m n}\right)
\end{array}\right]
$$

Make each particle generate a random number between [0,1], and compare it with the set mutation probability. If $r \leq p_{b}$, the probability amplitude of randomly selected $[i / 2]$ qubits is changed by quantum non gate, where $i$ is the total number of qubits.

\subsection{Solution process}

The specific optimization solution flow of economic operation of IES based on 
MCS-QPSO algorithm is shown in Fig.5.

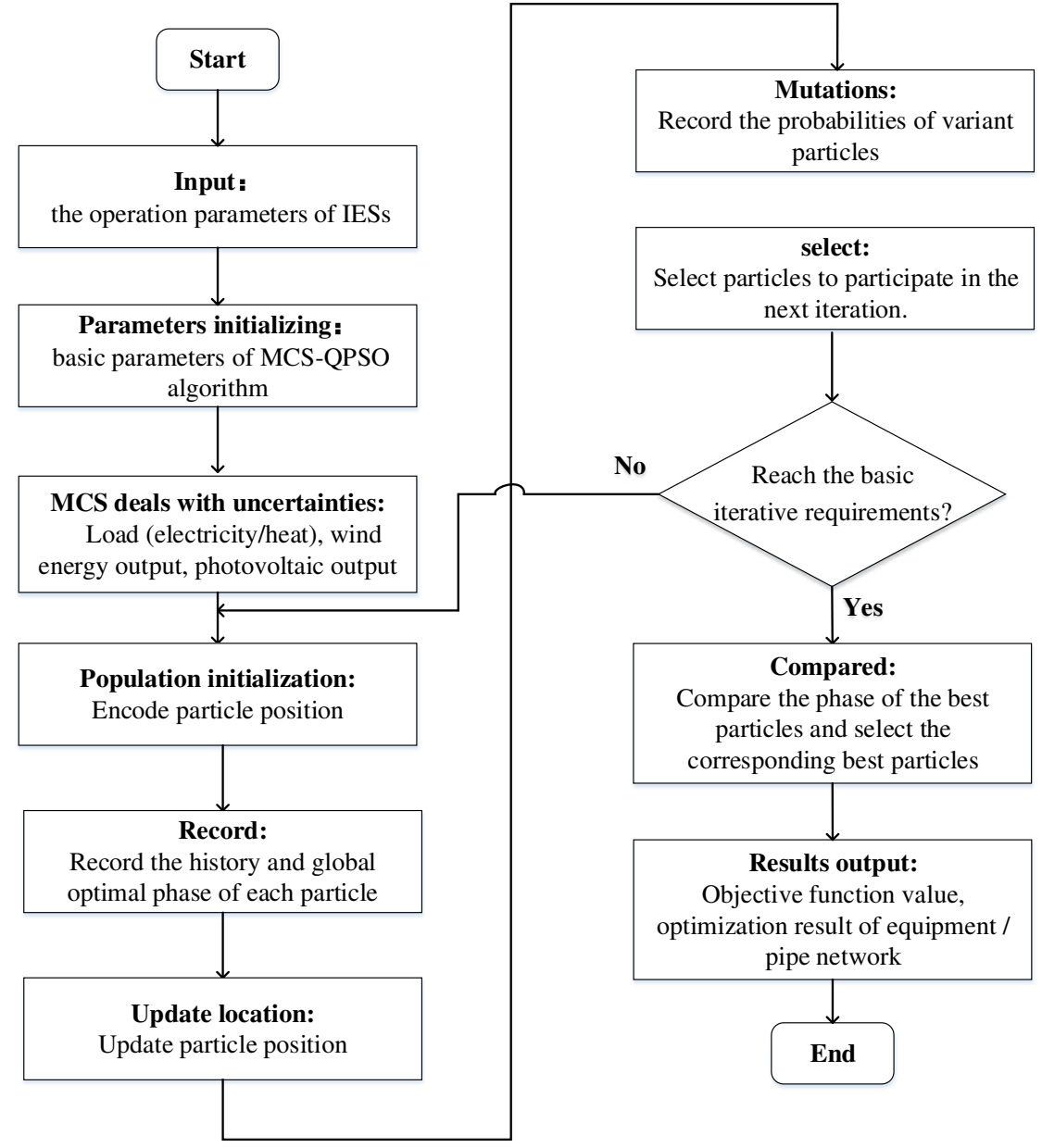

Fig.5. Optimization process of IES economic operation based on MCS-QPSO algorithm

(1) Initialization model and algorithm parameters. According to the historical load data, the initial load value of each load node and the normal distribution of operation scheduling level year are determined. According to the historical data of wind speed, the parameters of Weibull distribution of the daily wind speed of IES are determined. According to the historical data of sunlight, the parameters of beta distribution of sunlight intensity in IES were determined.

(2) Distributed energy output initialization. Based on the probability distribution of renewable resources, the power generation of renewable energy is predicted by using historical data.

(3) Constraint check. The constraints of access scheme are verified by Monte Carlo simulation. If the condition is met, perform step (4), otherwise return to step (2).

(4) Solution space transformation and fitness calculation. According to equation (28), the solution space is transformed, and the randomly generated particle position is mapped to the solution space of the problem. According to the objective function formula (14) and the corresponding constraints, the fitness of each particle is calculated, and the individual extreme point and the global extreme point are found. If it is better than the historical extremum, the current extremum is used to replace the 
historical extremum.

(5) Particle state update and mutation operation. According to equations (29) and (30), the particle state update and mutation operation are realized.

(6) Cycle operation. Return to step (2) to cycle until the convergence condition is met or the maximum number of iterations is reached.

\section{Case study}

\subsection{Background}

The structure of the 36-node heating network is shown in Fig.6. Among them, the electric and heat coupled devices include: CHP located at nodes 1, 13, 17 of the heat network, and an electric boiler (EB) located at node 32 of the heat network.

The total installed capacities of CHP and electric boilers are $70 \mathrm{MW}$ and $90 \mathrm{MW}$, respectively. Since the time scale of the dynamic process of electricity transmission is much smaller than that of the heat grid, the grid structure is ignored in this paper. In order to prove the validity of this method more clearly in this paper, the example is constructed to compare two typical scenarios:

Scenario 1: Electro-heat IES operation optimization model without considering the dynamic properties of heat energy transfer.

Scenario 2: Electro-heat IES operation optimization model considering dynamic properties of heat energy transfer.

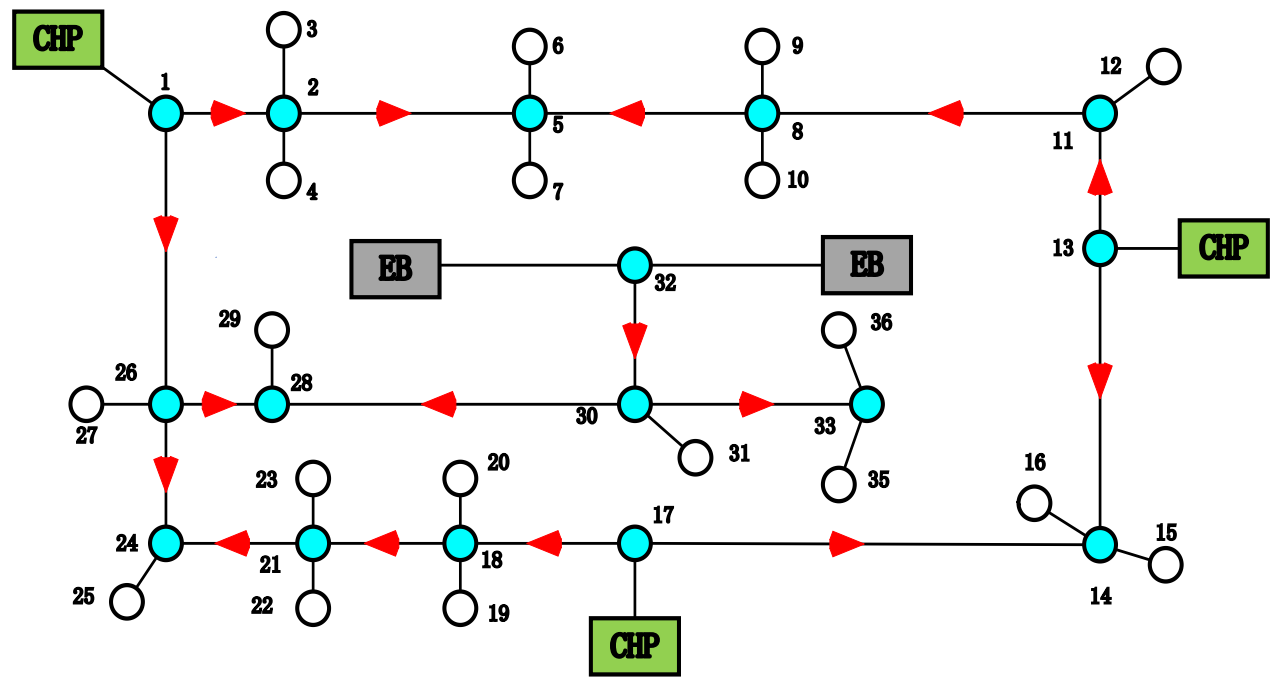

Fig. 6. 36-node heating network structure

The heat output of the energy equipment in Scenario 1 should be equal to the heating demand at all times, regardless of the latency and virtual energy storage characteristics of the heat network, and only the losses of the heat network should be considered. In this scenario, the return water temperature in the first heat exchange station is kept constant, thus ensuring that the heat output of the source-side energy conversion equipment is equal to the charge-side heat demand at each point in time. 
In order to respond to the demand response policy in the park, the park needs to optimize scheduling through peak cutting and valley filling, peak shifting operation, etc. The electricity price, estimated power consumption and heat load of a typical day in the park are shown in Fig.7.

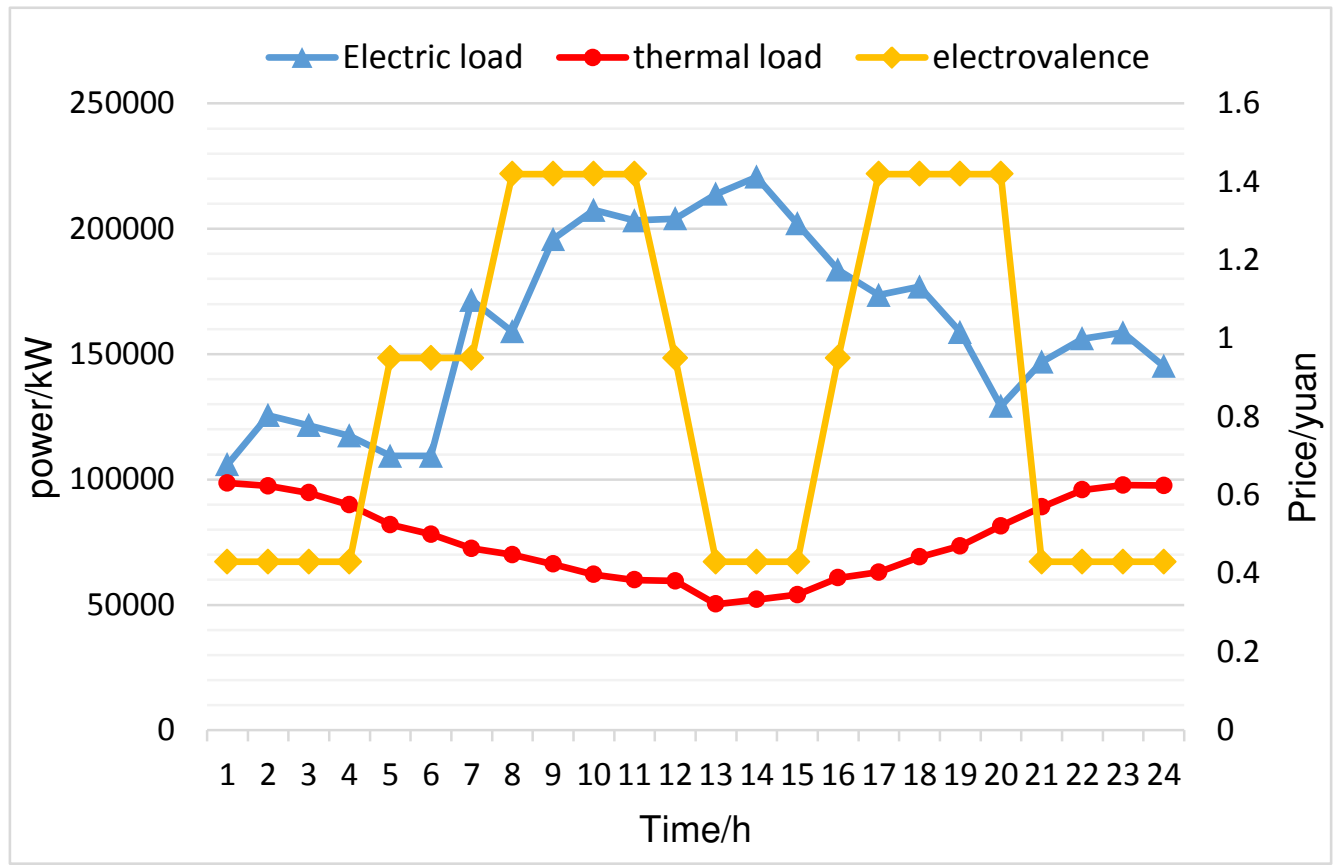

Fig.7. Hourly tariff and typical daily electricity load, heat load

\subsection{Simulation results}

\section{(1) Heat system output comparison}

Fig.9, Fig.10 are the results of heat system output under two scenarios. As can be seen from the figures, the heat source heat supply and heat load of the heat exchange station in the heat system in scenario 2 are not synchronized, while scenario 1 is synchronized.

The dynamic properties of heat transfer in Scenario 2 are manifested in the heat system in two ways:

1) At the tariff valley moment, the electric boiler first satisfies the heat load demand, and then exerts more force within the constraints of the virtual energy storage characteristics of the heat network, so as to realize the heat storage of the virtual energy storage of the heat network; then at the tariff level and peak moment, the heat energy stored in the heat network in advance is released to satisfy part of the heat load demand at that time. For example, at 14:00-15:00, the system heat output is significantly higher than the heat load demand, while at 16:00-17:00, the system heat output is significantly lower than the heat load demand.

2) The positive relationship between CHP loading rate and COP can be seen in Fig 8. Based on the time-delay and virtual energy storage characteristics of the heat network, it is firstly possible to increase the output force while the CHP meets the 
heat load demand, which not only improves the heat efficiency but also saves the operation cost of the CHP at the next moment. For example, in 1:00 4:00 and 21:00 24:00, the CHP in the heat system increases the output force at 1:00, 3:00, 21:00, 23:00, so that the CHP does not output at 2:00, 4:00, 22:00, 24:00; also, in the CHP continuous heat output period, the average COP of CHP can be increased through the CHP preheating output force, for example, in 18:00 20:00, the CHP preheating output force, so that the average COP of CHP at 18:00, 19:00, 20:00 three time points relative increases.

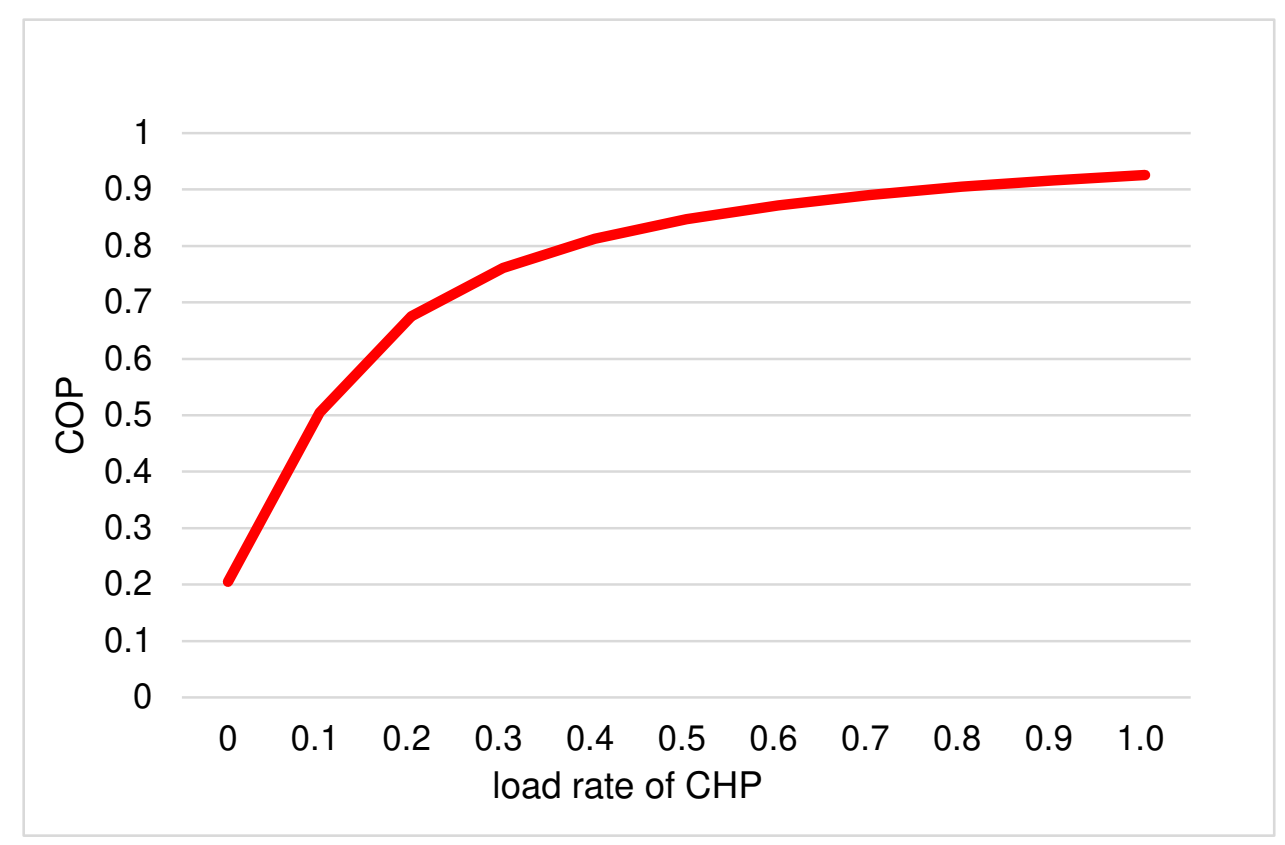

Fig.8. The relation curve between load rate and COP of CHP

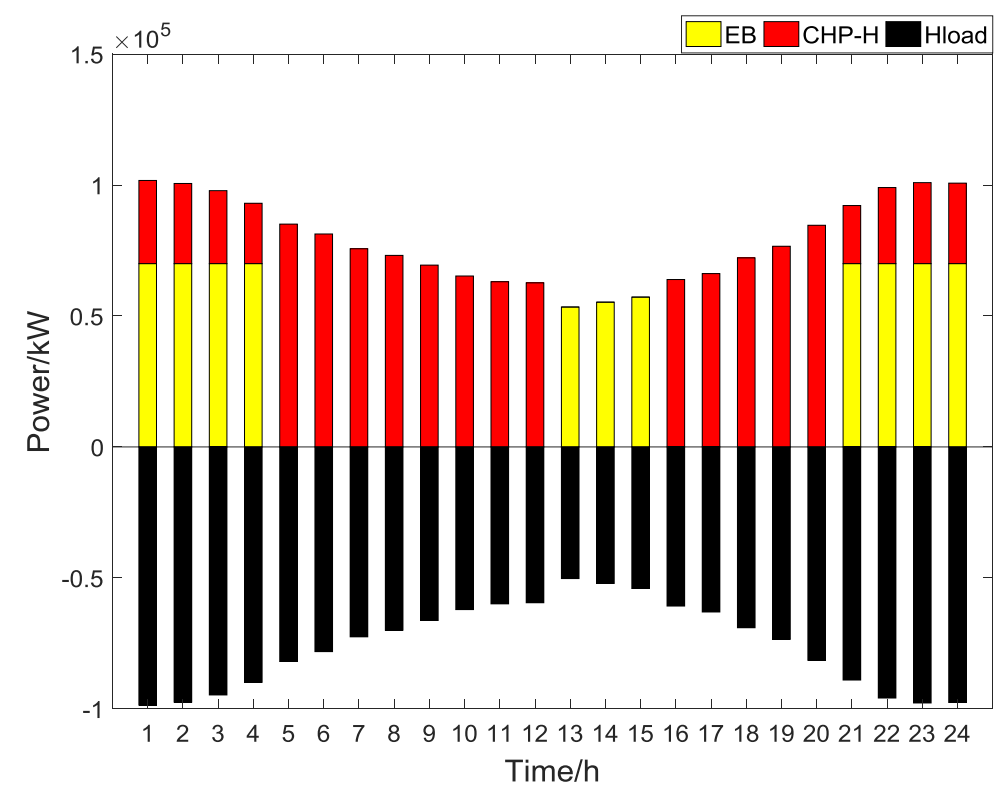

Fig.9. Scenario 1 optimal dispatch results of heat system 


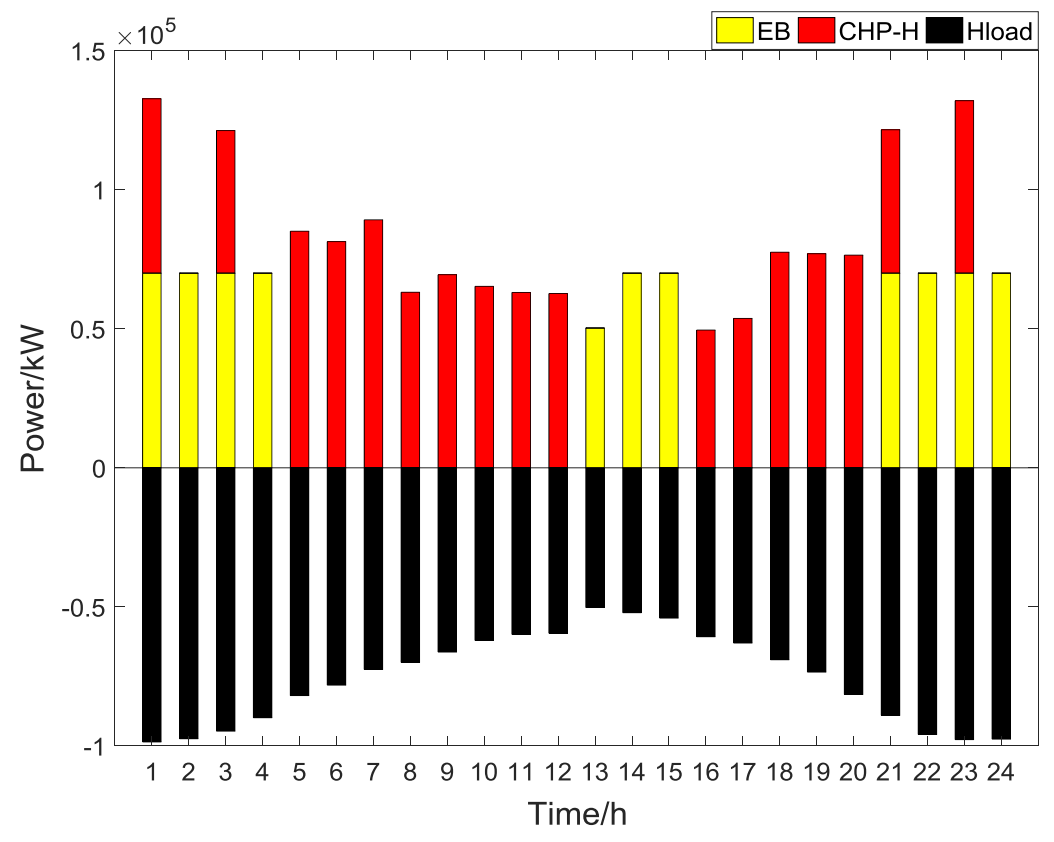

Fig.10. Scenario 2 optimal dispatch results of heat system

\section{(2) Electricity system output comparison}

Fig.11, Fig.12 show the results of the power system output for the two scenarios. Each power supply equipment will reasonably generate electricity during each time period to meet the demand of users and equipment operation. The difference in the electric system output in the two scenarios is synchronized with the heat system. There are two main reasons: first, because of the dynamic characteristics of heat energy transmission in scenario 2 , more electricity is purchased to achieve virtual heat storage during the trough of the electricity price, and electric heating is reduced during the price plateau and peak, and the electricity output in scenario 2 is higher than scenario 1 during the trough and lower than scenario 1 during the peak; second, CHP adopts the operation mode of "heat to power", because CHP considers the relationship between the load rate and the COP in the heat system, CHP preempts to increase the average COP of CHP and reduce operation cost, so CHP in the electric system and the heat system synchronize. 


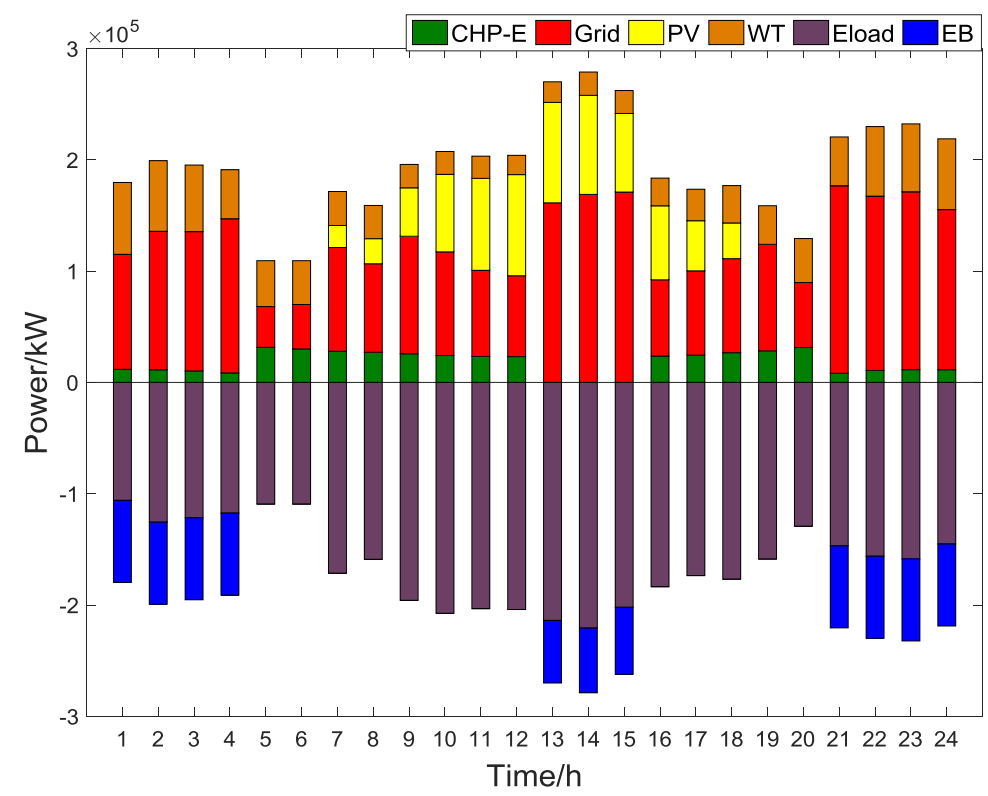

Fig.11. Scenario 1 optimal dispatch results of electric system

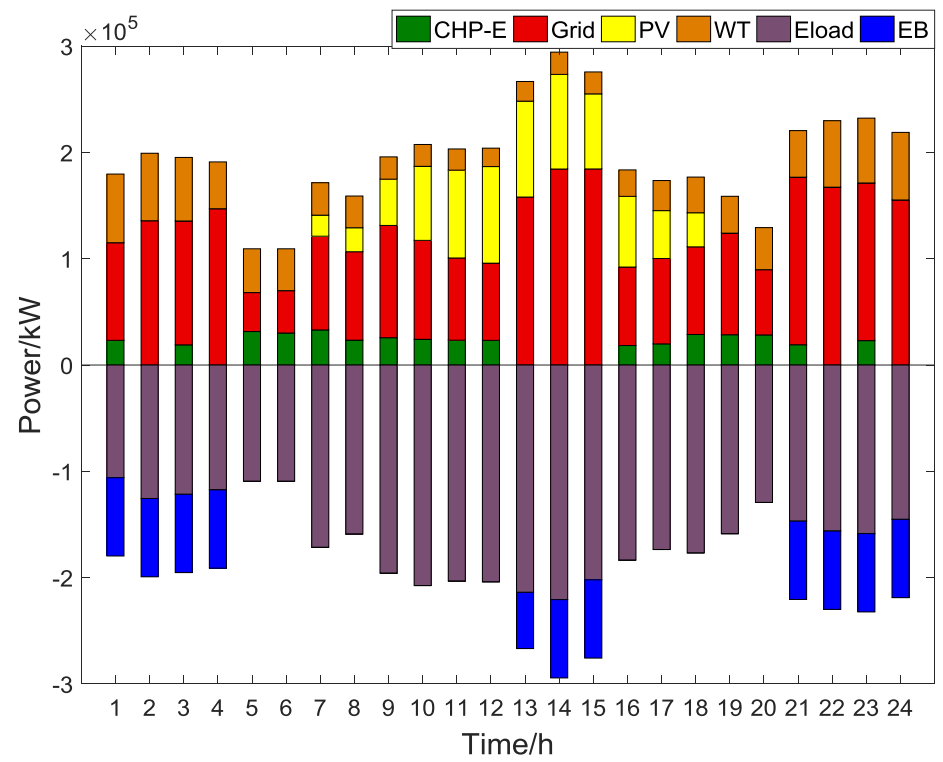

Fig.12. Scenario 2 optimal dispatch results of electric system

\subsection{Discussion and analysis}

\section{(1) Comparison of energy consumption for different scenarios}

The power consumption of electric boiler and system gas consumption of the two scenarios are shown in Fig.13. In valley electricity price, in scenario 2, if the electric boiler has space for virtual energy storage, the electric boiler will increase its output, and the power purchase will increase accordingly; if the electric boiler does not have space for virtual energy storage, and because in both scenarios, the electric boiler will give priority to output at that time, which also leads to the same power purchase situation in the figure. In flat and peak electricity price, due to the high cost of power purchase, CHP gives priority to output. Because CHP can meet the demand of heat 
load in the park, EB output is not required. Thus, the power consumption of electric boiler of scenario 2 is higher than that of scenario 1 .

As can be seen from Fig.13, the gas consumption of the system of scenario 2 is significantly lower than that of scenario 1 . There are two reasons for this: first, the electric boiler purchases electricity in the valley time, realizing the virtual heat storage; second, the CHP pre output increases its COP.

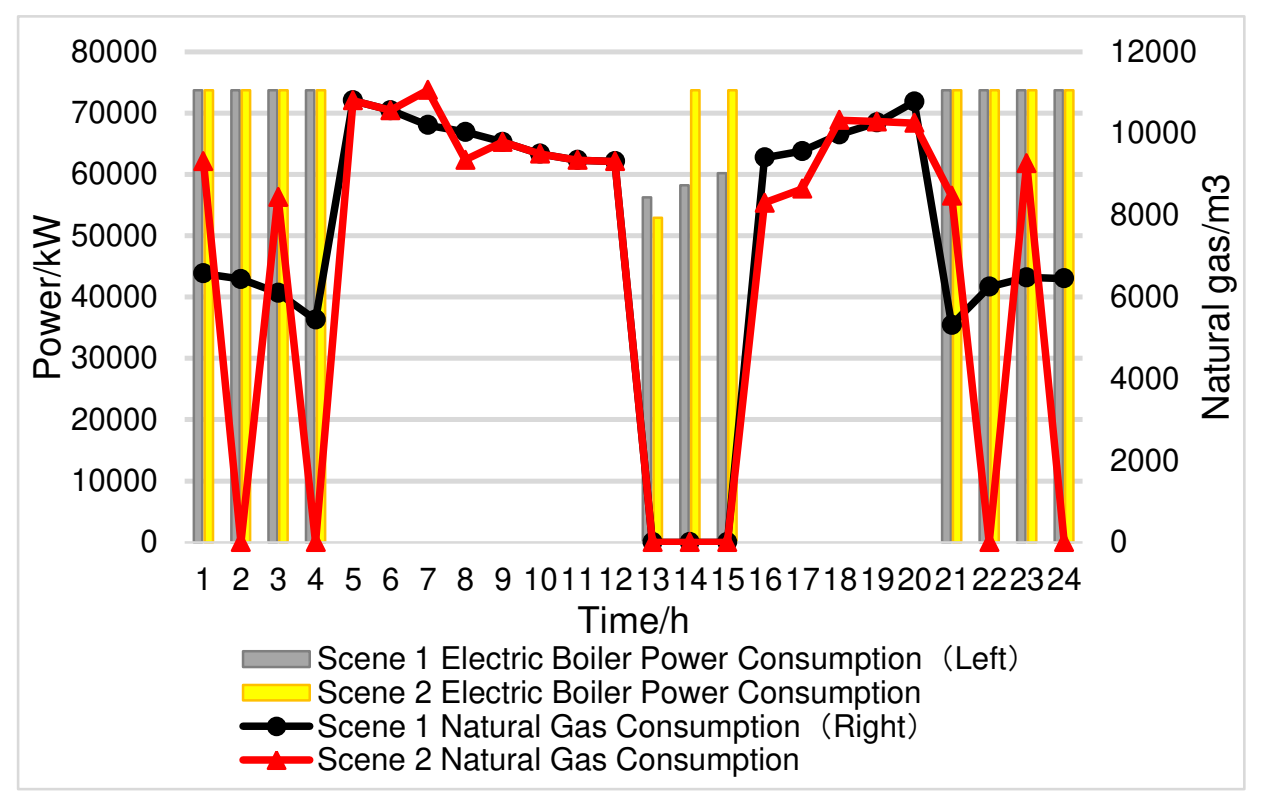

Fig.13. Electricity Consumption of Electric Boilers and Natural Gas Consumption of the System in Two Scenarios

\section{(2) Cost comparison of different scenarios}

Based on the conclusion in Fig.11, although the electricity purchase of electric boiler in Scenario 2 is higher than that of Scenario 1, the gas purchase of Scenario 2 is much lower than that of Scenario 1. By comparing the energy cost of the two scenarios in Fig.14 and Tab.2, the electricity purchase cost of Scenario 1 is lower than that of Scenario 2, the gas purchase cost of Scenario 2 is lower than that of Scenario 1, and the total operation cost of Scenario 2 is lower than that of Scenario 1. Therefore, the advantages of the coordination and complementarity of electric and heat systems are finally reflected by gas purchase cost. 


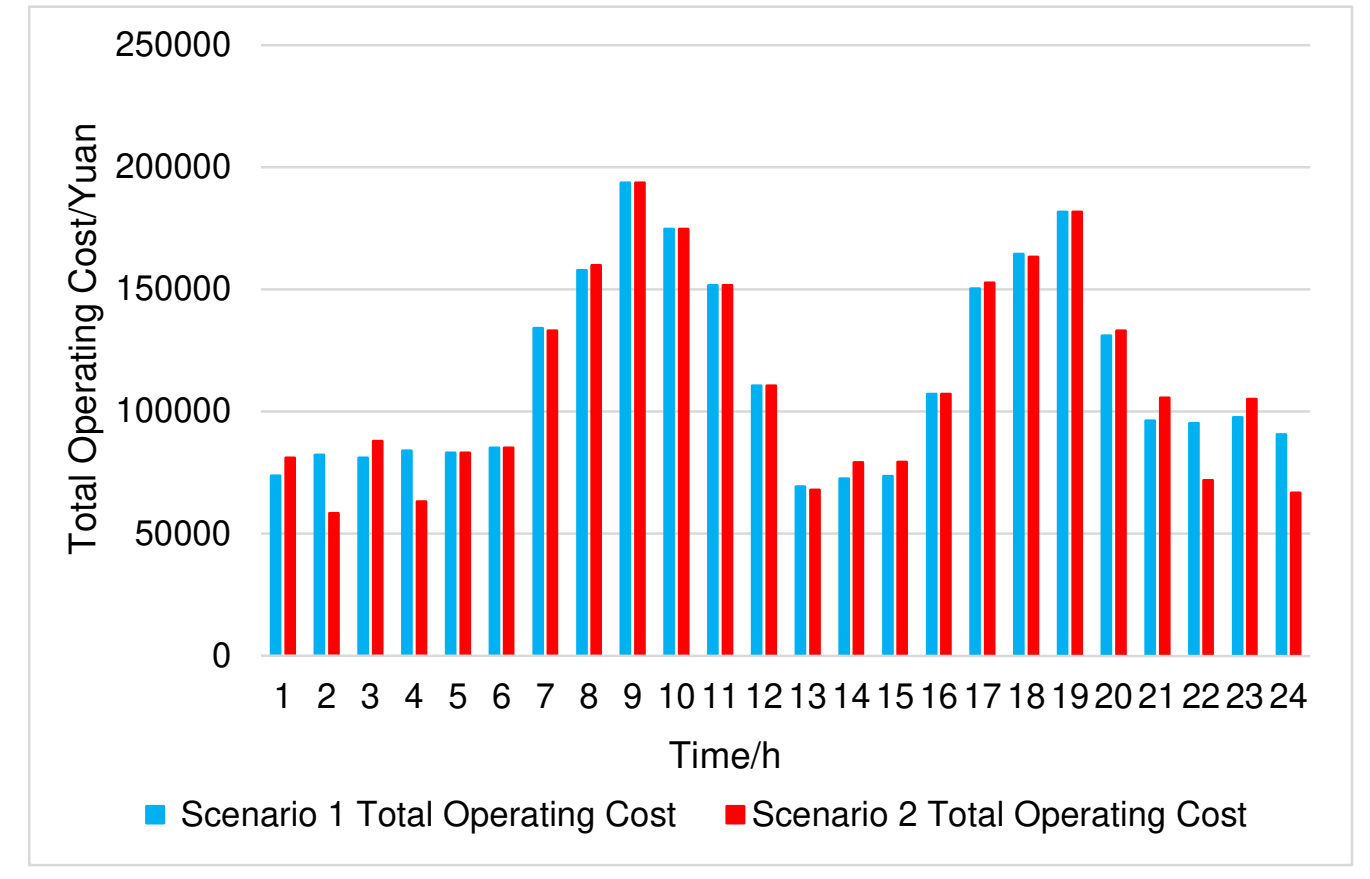

Fig.14. System Heat Output and Total Operation Cost for Two Scenarios

Tab.2 Energy Consumption Cost in Two Scenarios

\begin{tabular}{|c|c|c|c|c|}
\hline Scenario & $\begin{array}{c}\text { Electricity } \\
\text { purchase Cost of } \\
\text { load } \\
\text { (Yuan) }\end{array}$ & $\begin{array}{c}\text { Electricity } \\
\text { Purchase Cost of } \\
\text { Electric Boilers } \\
\text { (Yuan) }\end{array}$ & $\begin{array}{c}\text { Gas } \\
\text { Consumption } \\
\text { Cost } \\
\text { (Yuan) }\end{array}$ & $\begin{array}{l}\text { Total Operation } \\
\text { Cost } \\
\text { (Yuan) }\end{array}$ \\
\hline Scenario1 & 1615402.024 & 328570.8335 & $\begin{array}{l}798343 . \\
2\end{array}$ & 2742316 \\
\hline Scenario2 & 1628590.117 & 339594.5704 & $\begin{array}{l}728855 . \\
2\end{array}$ & 2697040 \\
\hline
\end{tabular}

\section{(3) Algorithm validation}

Furthermore, the MCS-QPSO algorithm proposed in this paper has the advantages of high speed and precision in solving large-scale engineering problems such as integrated energy system optimization. This section compares the performance of MCS-QPSO algorithm and genetic algorithm (GA) in solving scenario 2. From Tab.3 and Fig.15, it can be seen that the operation result of MCS-QPSO algorithm is far better than that of GA. 


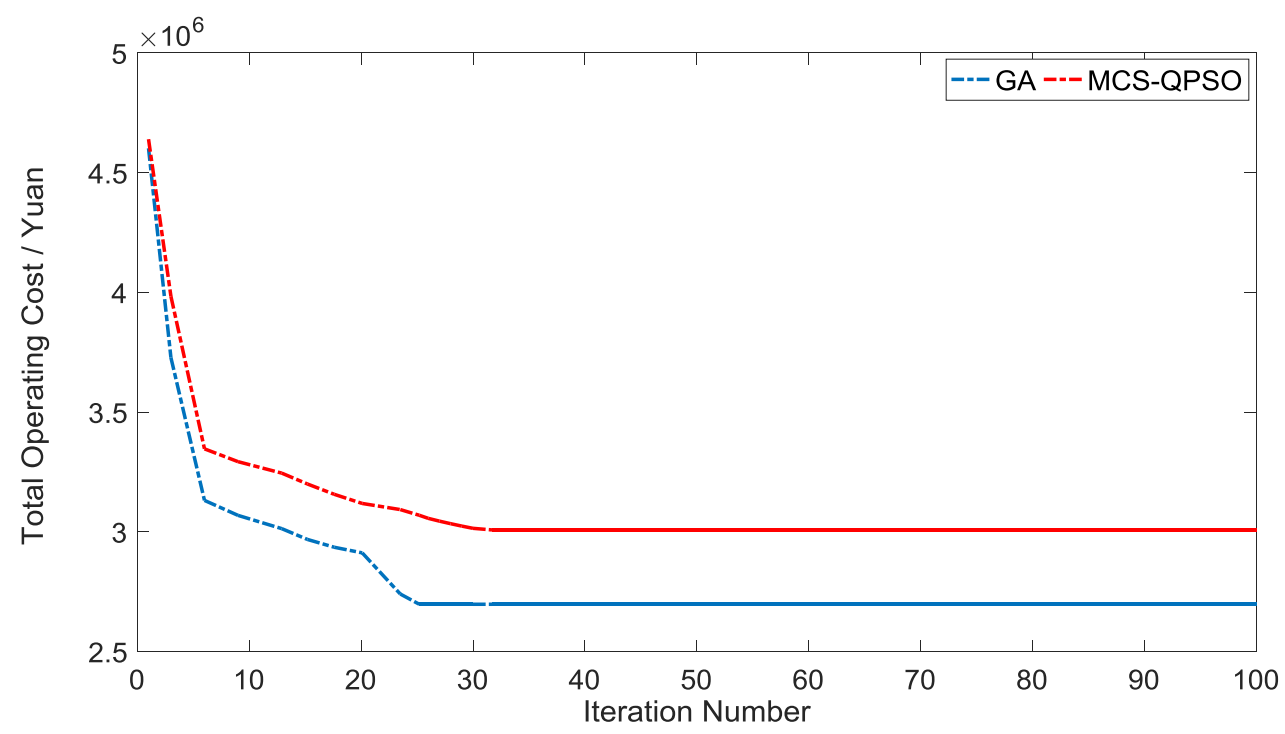

Fig.15. Comparison of iterative curves for different algorithms

Tab.3 Comparison of the performance of MCS-QPSO and GA

\begin{tabular}{ccccc}
\hline Algorithm & Best cost / yuan & Average cost / yuan & Iteration Number & Computing time/s \\
\hline GA & 3007507 & 3058797 & 31.7 & 32.33 \\
MCS-QPSO & 2697040 & 2753769 & 25.2 & 29.89 \\
\hline
\end{tabular}

In addition, the MCS-QPSO algorithm outperforms the GA in terms of calculation results and returns. MCS-QPSO algorithm makes use of the characteristics of Monte Carlo and quantum gate to enhance the global search ability and get better results.

\section{Conclusion}

In this paper, based on the latency and virtual energy storage characteristics of the heating network, an operation optimization model for the electric-heat IES is established, which takes into account the dynamic characteristics of heat energy transmission:

(1) Analyzed and characterized the latency of energy transfer in the heat network and the ambiguity of the user's physical senses;

(2) Establishment of a dynamic model of heat energy transfer and a dynamic model of heat network nodes and analysis of the virtual energy storage characteristics of heat networks;

(3) Construct an electro-heat IES operation optimization model that takes into account the virtual energy storage characteristics of the heat network.

Simulation results show that virtual energy storage through the preemptive power of heating equipment not only responds to local time-sharing electricity prices but also saves gas consumption. This achieves a coordinated complementarity 
between the electric and heat systems in time and space, thus reducing the total operation cost of the system. The next step can be to consider the combination of the three energy conversion device models in IES, to consider building a dynamic model of the energy transfer dynamics of the cold-heat-electric network, and to explore the coordinated complementarity of the cold-heat-electric system in the space-time range.

\section{Declarations}

\section{Acknowledgements:}

The authors would like to thank the three anonymous reviewers for their helpful comments and suggestions.

Funding for this research was provided by "Central University Basic Research Service Fee" (2018ZD13), "111 Project" (B18021) and "Beijing Social Science Fund Project" (18GL042), and is gratefully acknowledged.

\section{Authors' contributions :}

Ersheng PAN is dedicated to the design of article structure, query and management of related literature. Hui LI is devoted to the application of model construction and solution methods. Zhidong WANG is committed to data acquisition, data analysis and interpretation. Dong PENG is devoted to the application of model construction and solution methods. Lang ZHAO is dedicated to work design, data acquisition, data analysis and interpretation. Yongli WANG is devoted to model building and software platform operation research. Feifei HUANG is devoted to model building and software platform operation research. Yang MA is devoted to model building and software platform operation research

\section{Funding:}

Funding for this research was provided by "Central University Basic Research Service Fee" (2018ZD13), "111 Project" (B18021) and "Beijing Social Science Fund Project" (18GL042).

\section{Availability of data and materials:}

The datasets used and/or analyzed during the current study are available from the corresponding author on reasonable request.

\section{Ethics approval and consent to participate:}

Not applicable.

\section{Consent for publication:}

Not applicable.

\section{Competing interests:}

The authors declare that they have no competing interests.

\section{Author details :}


1. State Grid Beijing Economic Research Institute, Beijing 102209, China; 2. School of Economics and Management North China Electric Power University, Changping District, Beijing 102206 China.

\section{References}

[1] Yu Xiaodan, Xu Xiandong, Chen Shuoyi, et al. A brief review to integrated energy system and energy internet[J]. Transactions of China Electrotechnical Society, 2016, 31(1): 1-13 .

[2] Stanislav P, Bryan K, TihomirR M. Smart grids better with integrated energy system[C]//Proceedings of the Electric Power \& Energy Conference (EPEC). Montreal, Canada, 2009.

[3] National Development and Reform Commission, National Energy Administration. Implementation Opinions on Promoting the Construction of Multi-energy Complementary Integration Optimization Demonstration Project [R]. Beijing: National Development and Reform Commission, National Energy Administration, 2016.

[4] Sun Hongbin, Guo Qinglai, Pan Zhaoguang, et al. Energy internet: concepet, architecture and frontier outlook $[\mathrm{J}]$. Automation of Electric Power Systems, 2015, 39(19): 1-8 .

[5] Yongli Wang, Yujing Huang, Yudong Wang, Haiyang Yu, et al. Optimal Scheduling of the Regional Integrated Energy System considering economy and environment[J], IEEE Transactions on Sustainable Energy, 2018, PP(99):1-1.

[6] Yang Shuai, Chen Lei, Xu Fei, et al. Optimal Dispatching Model for Abandoning Wind and Consuming Water in Electric Heating Integrated Energy System Based on Energy Flow [J]. Power Grid Technology, 2018, 042(002):417-425.

[7] Zhang Gang, Zhang Feng, Zhang Li, et al. Distributed Calculation Method for Power Flow of Electric-Heat Combined System Considering Multiple Coupling Units, Journal of China Electric Engineering, 2018, 038(022):6594-6604.

[8] Qun Chen, Junhong Hao, Lei Chen, et al. Global Energy Transport Model in Electric-Heat Integrated Energy System [J]. Automation of Electric Power Systems, 2017, 41 (13): 7-13.

[9] Zheng Chaoming, Huang Bonan, Wang Zixin, et al. Multi-objective Optimal Dispatch of Electric Heating Integrated Energy System Considering Network Transmission Loss [J]. Power Grid Technology, 2020, 44(1).

[10] Zhang D, Evangelisti S, Lettieri P, Papageorgiou L G. Optimal design of CHP-based microgrids: Multiobjective optimisation and life cycle assessment[J]. Energy, 2015, 85:181-193. 
[11] Chen Qun, Hao Junhong, Chen Lei, et al. Overall Energy Transport in Electric-Heat Integrated Energy SystemModel [J]. Power System Automation, 2010,17,41 (13): 7-13.d O I: 10.7 500/AEPS20161209002.

[12] Chen Haoyong, Wen Junzhong, Wang Zengyu, et al. Transfer laws and equations of energy networks[J]. Journal of Xi'an Jiaotong University, 2014, 48(10): 66-76 .

[13] Liu X, Jenkins $\mathrm{N}$, Wu J, et al. Combined analysis of electricity and heat networks[J]. Energy Procedia, 2014(61): 155-159.

[14] Liu X. Combined analysis of electricity and heat networks[D]. Cardiff: Cardiff University, 2014.

[15] Molitor, ChristophMarin, MilahiHernández, et al. Decentralized coordination of the operation of residential heating units[C]// 2014.

[16] Pei Wei, Deng Wei, Shen Ziqi, et al. Energy coordination and optimization of hybrid microgrid based on renewable energy and chp supply[J]. Automation of Electric Power Systems, 2014, 38(16): 9-15 .

[17] Jin Xiaolong, Mu Y unfei, Jia Hongjie, et al. Optimal scheduling method for a combined cooling, heating and power building microgrid considering virtual storage system at demand side[J]. Proceedings of the CSEE, 2017, 37(2): 581-591 .

[18] Jin Xiaolong, Mu Yunfei, Jia Hongjie, et al. Optimal scheduling method for a combined cooling, heating and power building microgrid considering virtual storage system at demand side[J]. Proceedings of the CSEE, 2017, 37(2): 581-591(in Chinese)

[19] Pei Wei, Deng Wei, Shen Ziqi, et al. Energy coordination and optimization of hybrid microgrid based on renewable energy and chp supply[J]. Automation of Electric Power Systems, 2014， 38(16): 9-15.

[20] He C, Dai C, Wu L, Liu T. Robust network hardening strategy for enhancing resilience of integrated electricity and natural gas distribution systems against natural disasters [J]. IEEE Transactions on Power Systems, 2018, 33(5):5758-5798.

[21] Zhang Qiong, Wang Liang, Xu Yujie, et al. Research progress in pumped heat electricity storage system: a review[J]. Proceedings of the CSEE, 2018, 38(1): $178-185,354$

[22] Liu Zifa, Zhang Wei, Wang Zeli. Optimal layout of urban electric vehicle charging stations based on quantum particle swarm optimization algorithm [J]. Proceedings of the CSEE, 2012, 32 (22): 39-45 
Figures

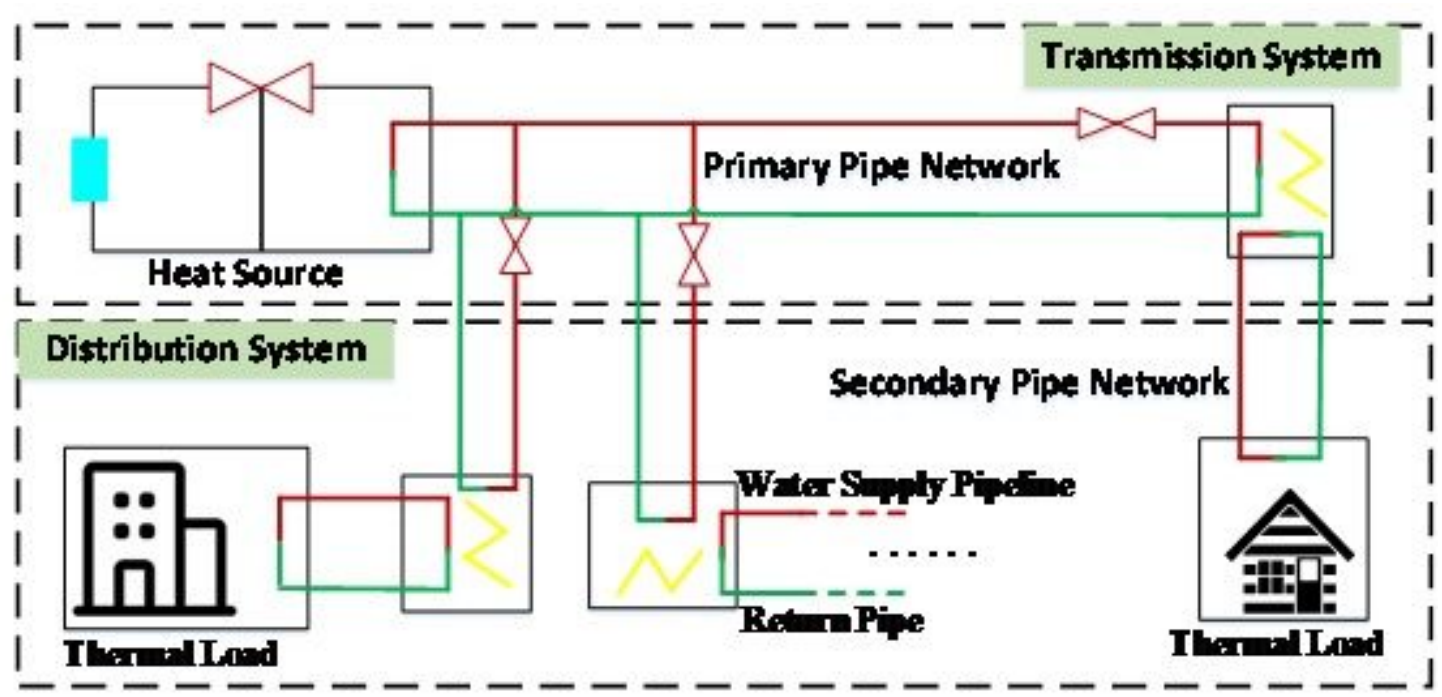

Figure 1

Architecture diagram of theheat system

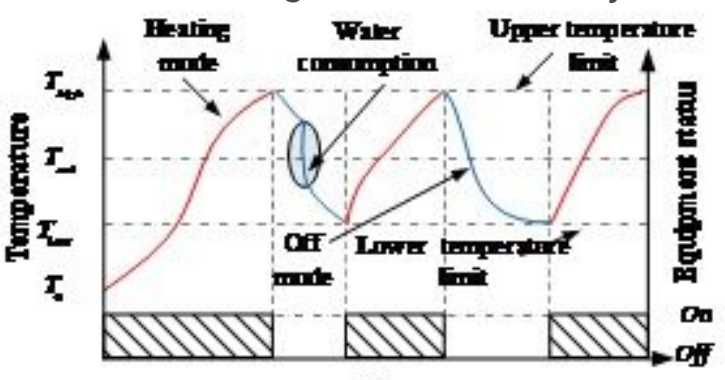

Tin

(a)

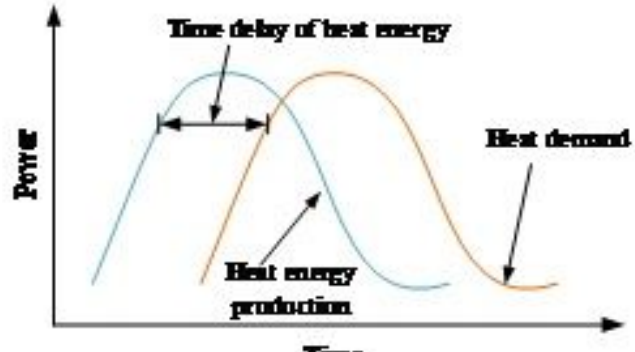

(b)

Figure 2

Characterization of the heat system

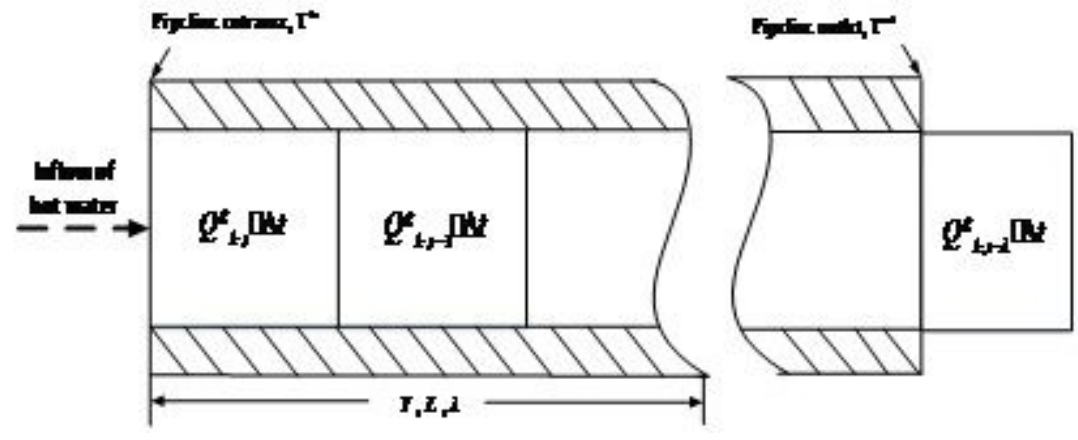

Figure 3

Cross-section of heating duct opening 


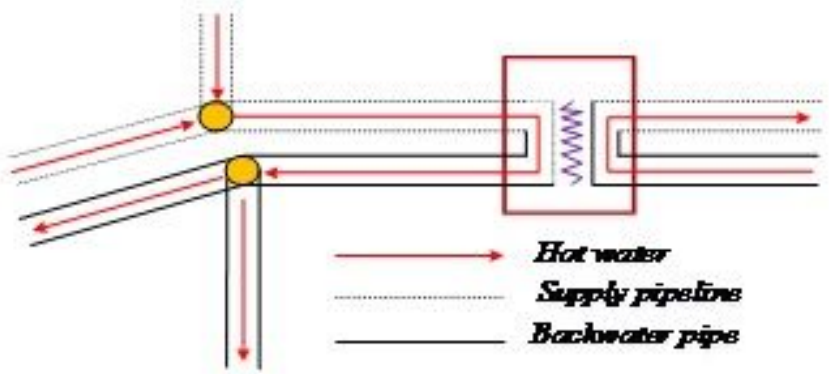

Figure 4

IES primary heat network structure diagram

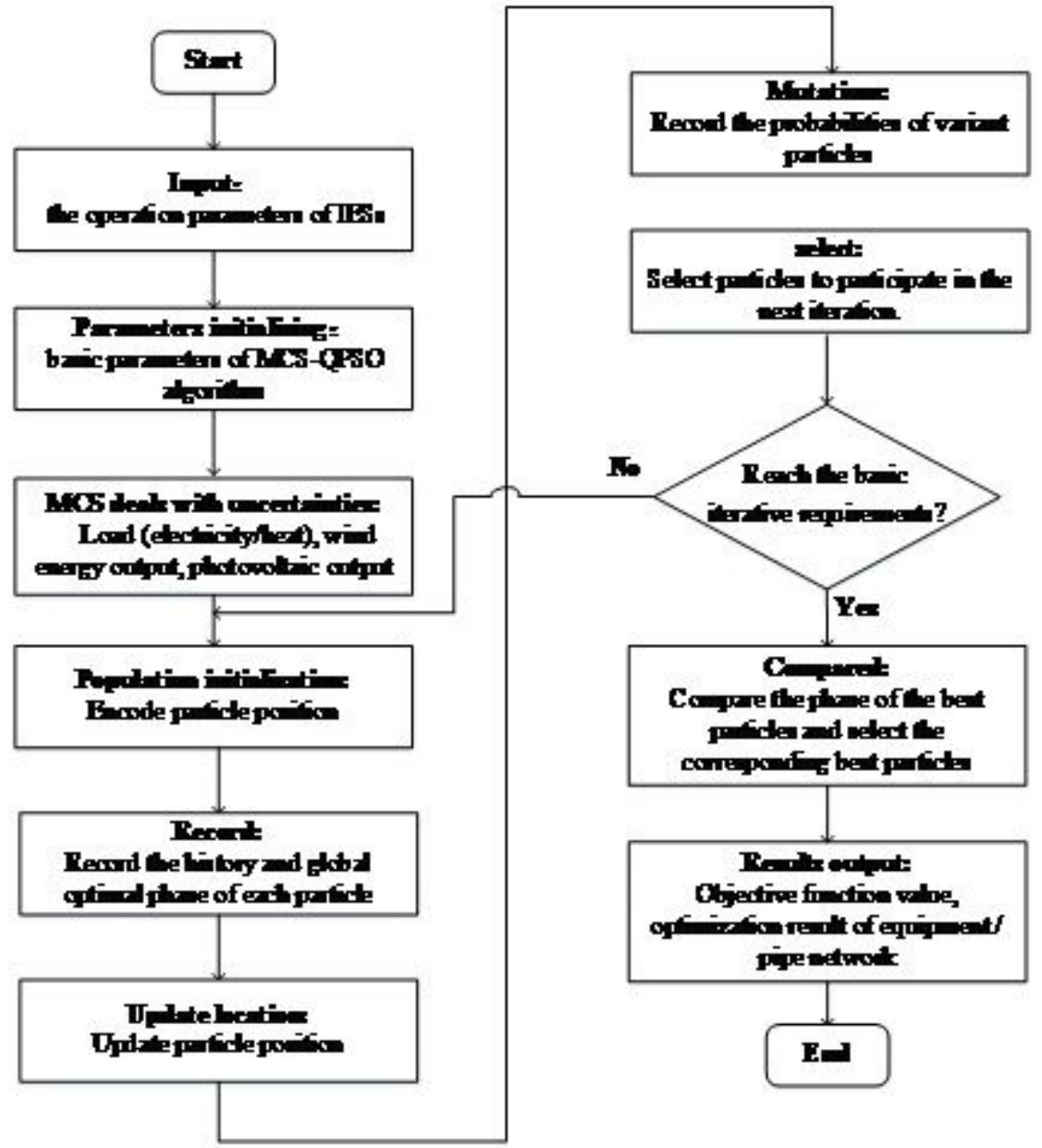

Figure 5

Optimization process of IES economic operation based on MCS-QPSO algorithm 


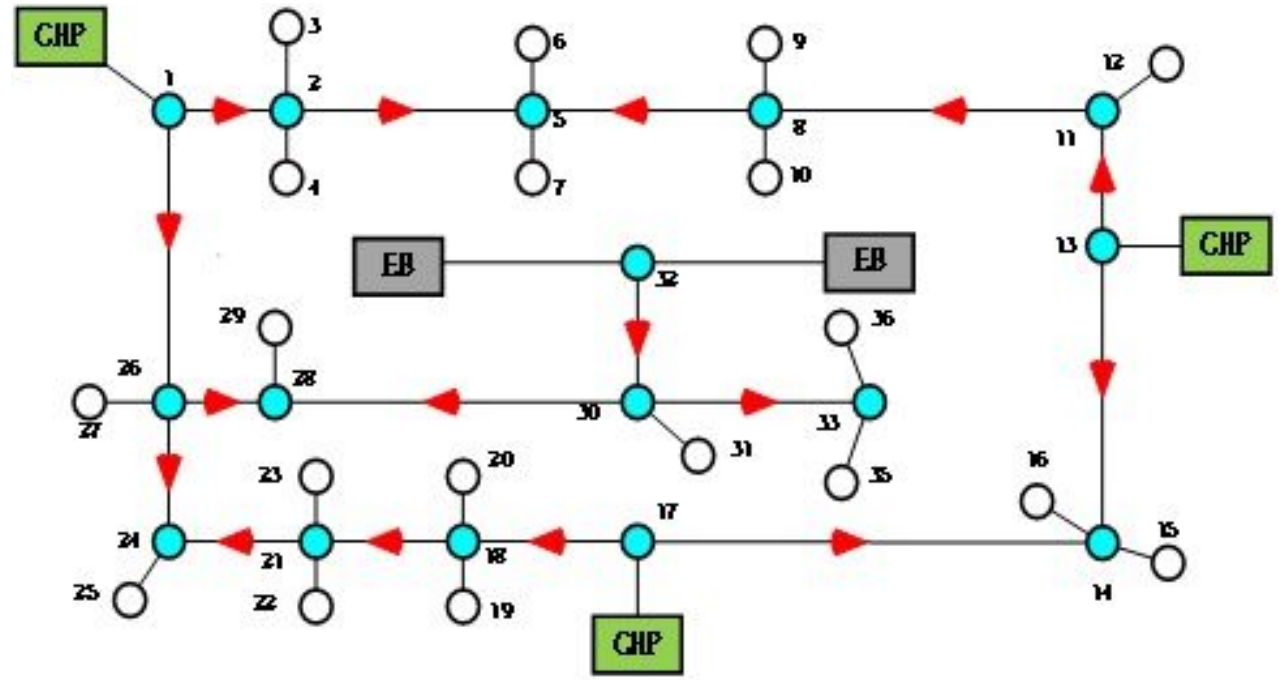

Figure 6

36-node heating network structure

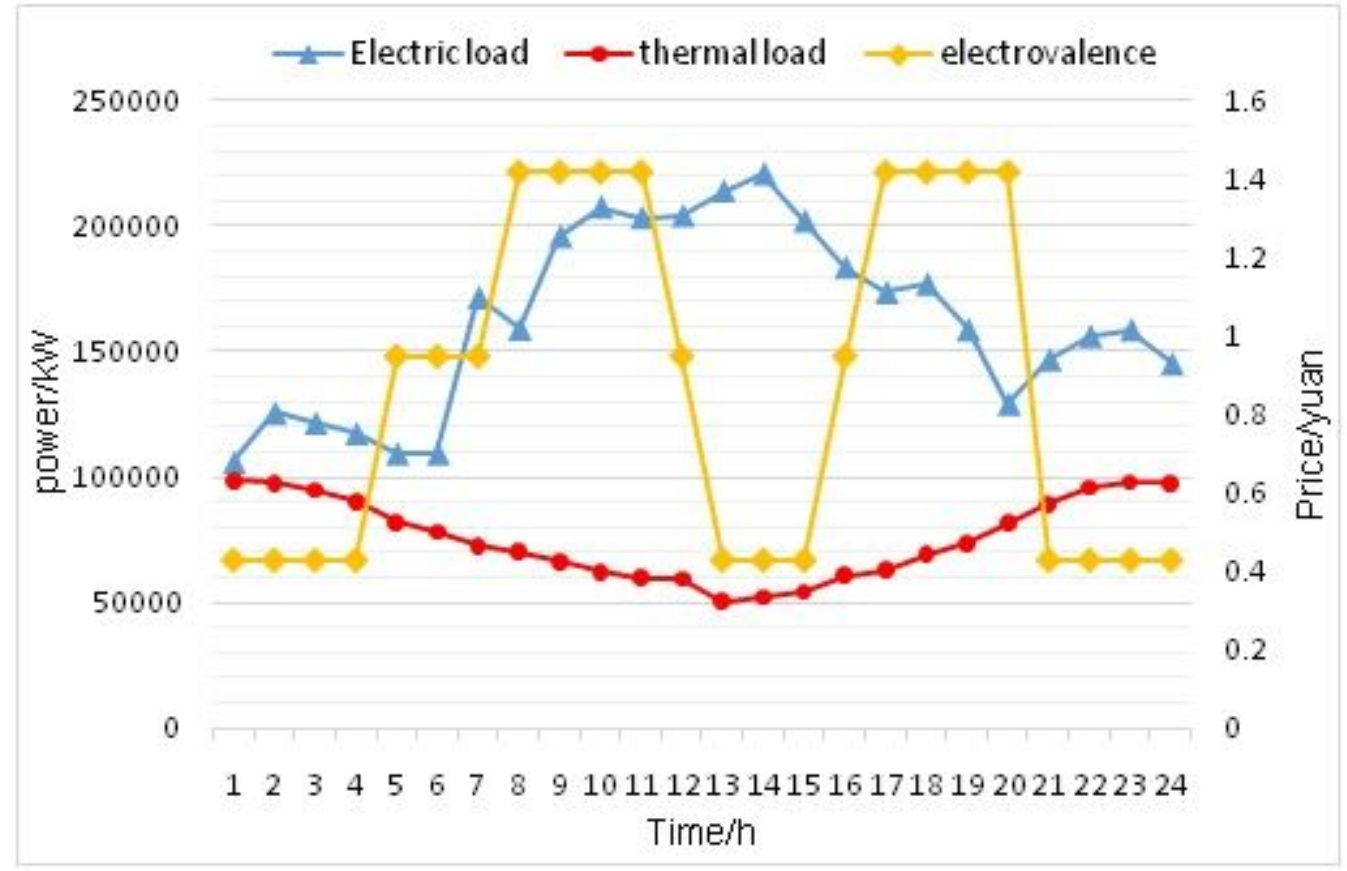

Figure 7

Hourly tariff and typical daily electricity load, heat load 


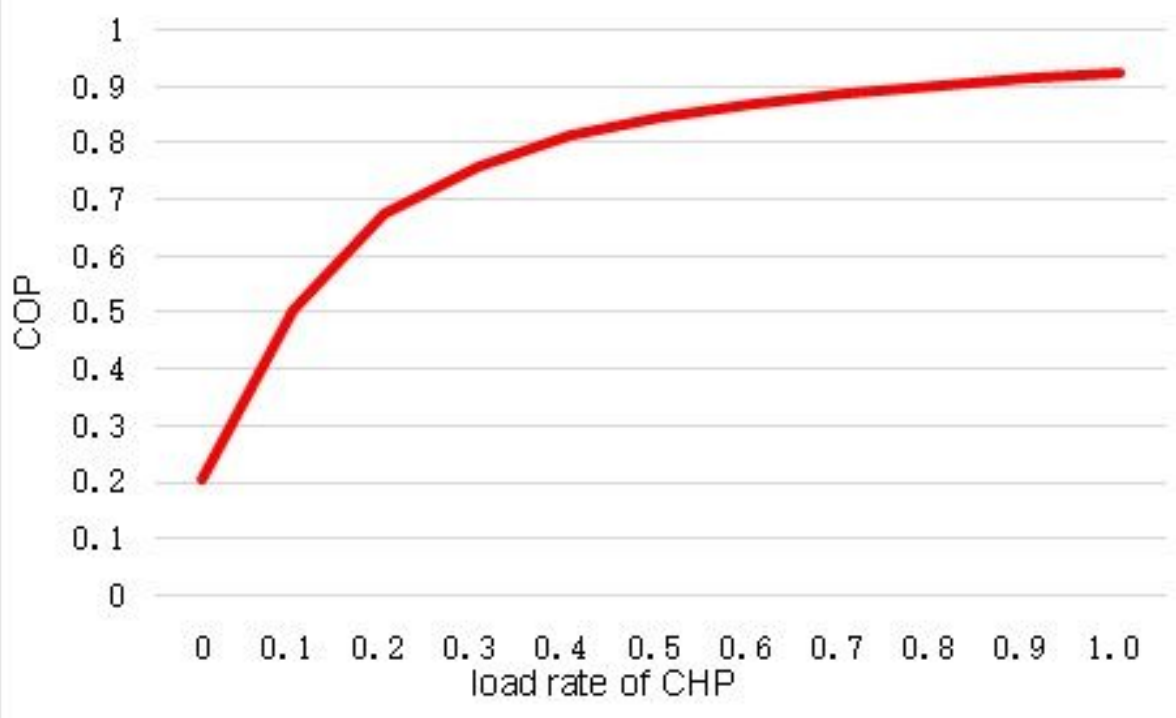

\section{Figure 8}

The relation curve between load rate and COP of CHP

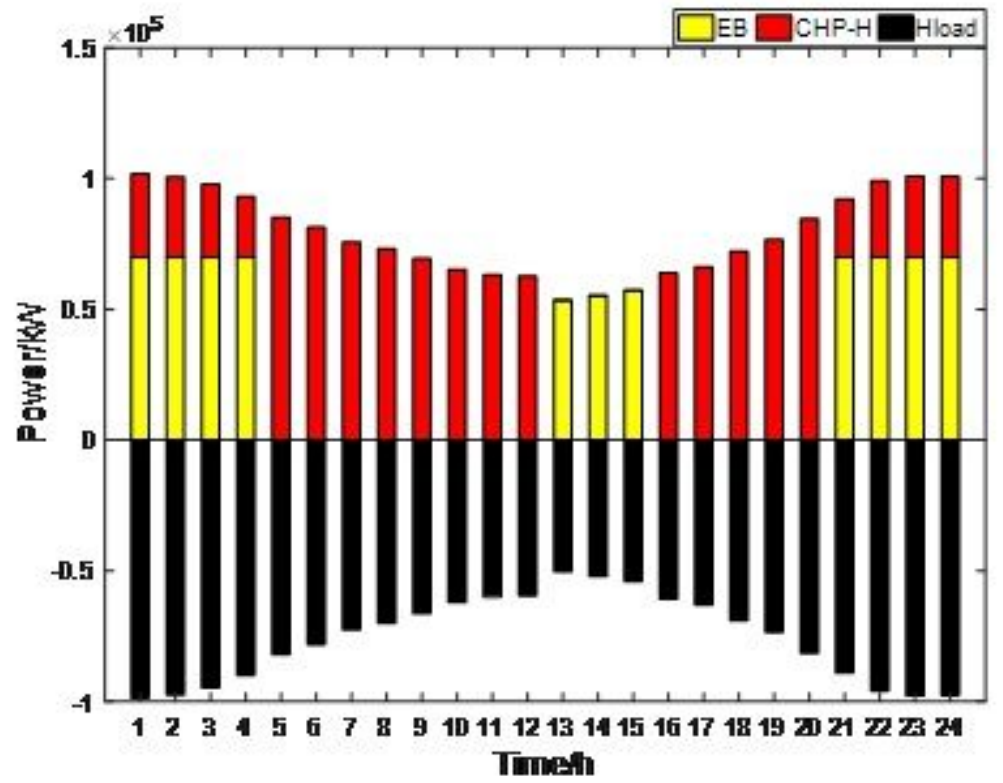

Figure 9

Scenario 1 optimal dispatch results of heat system 


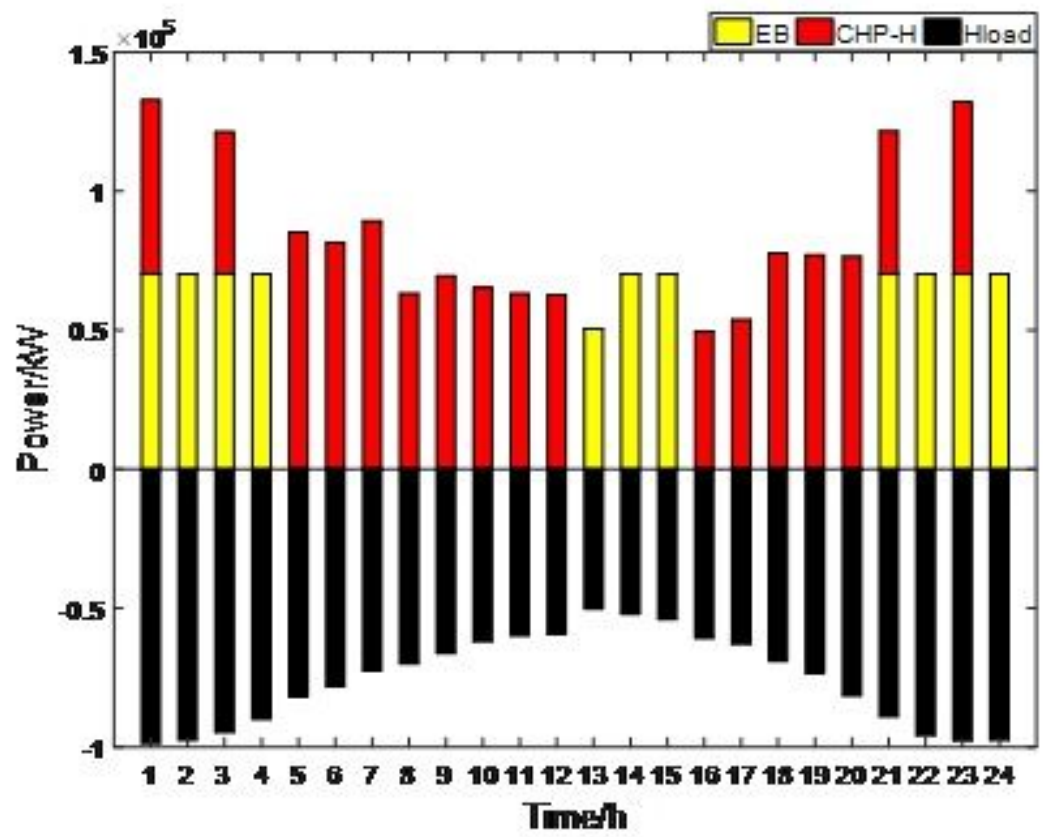

Figure 10

Scenario 2 optimal dispatch results of heat system

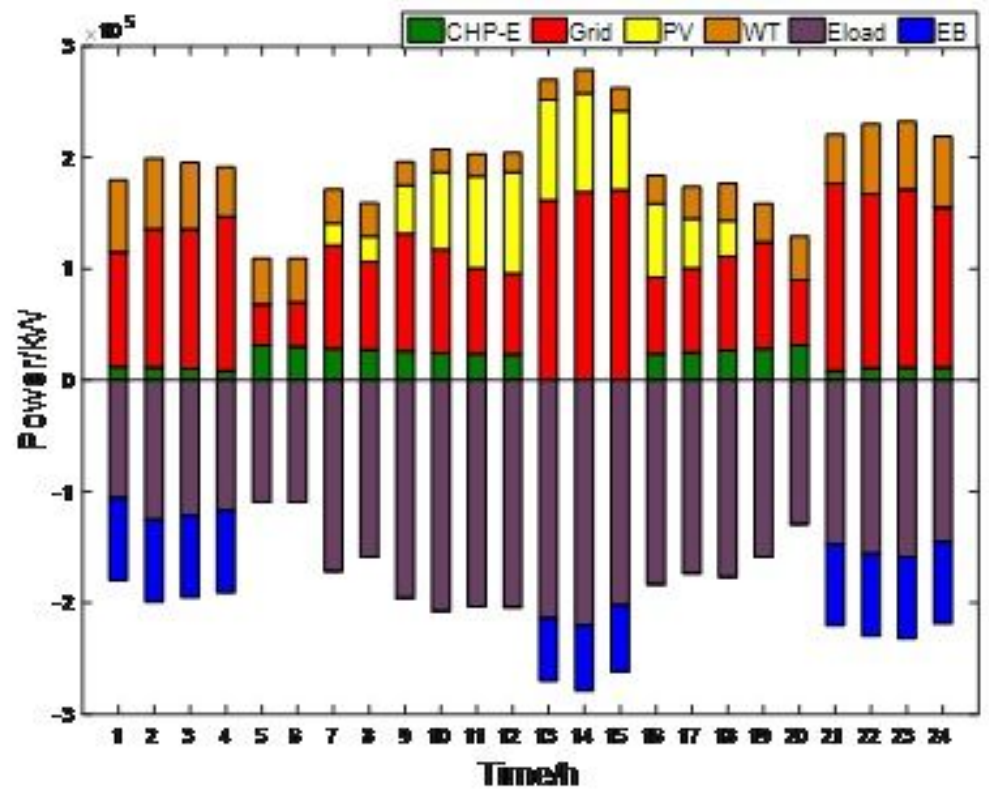

Figure 11

Scenario 1 optimal dispatch results of electric system 


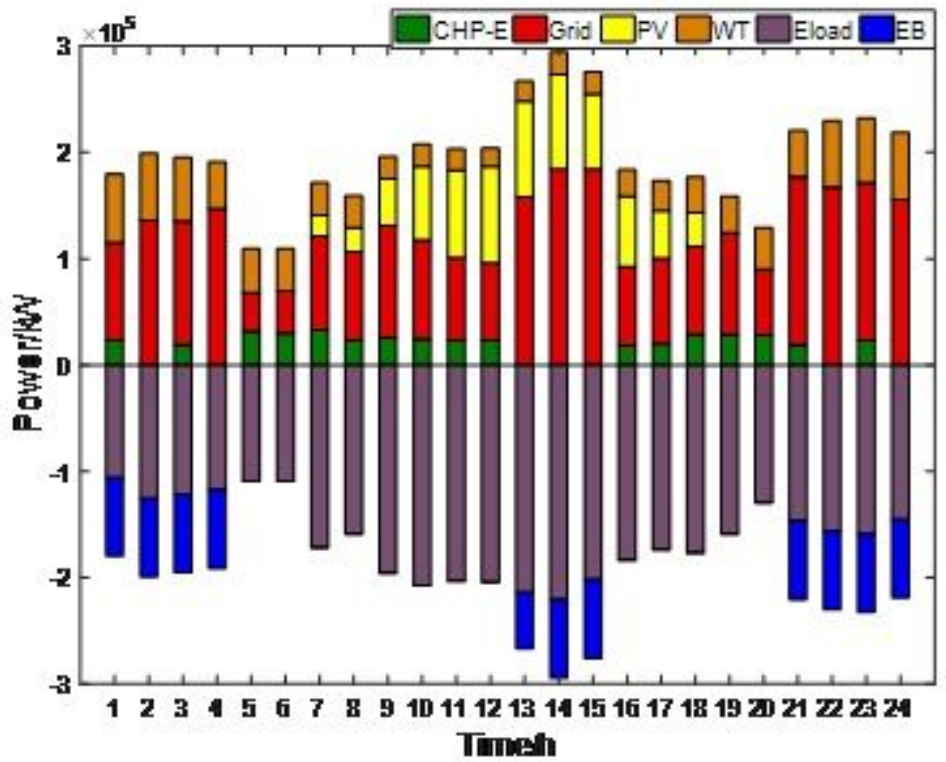

Figure 12

Scenario 2 optimal dispatch results of electric system

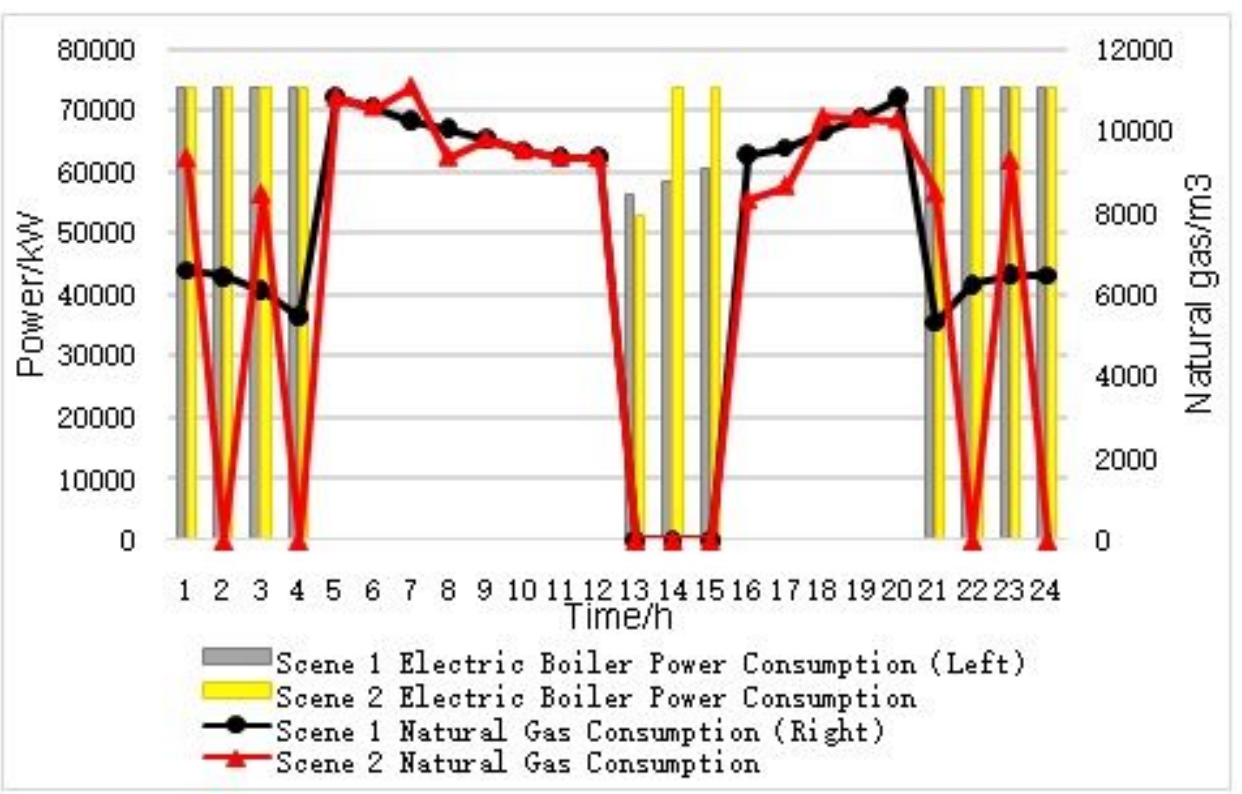

Figure 13

Electricity Consumption of Electric Boilers and Natural Gas Consumption of the System in Two Scenarios 


\section{0}

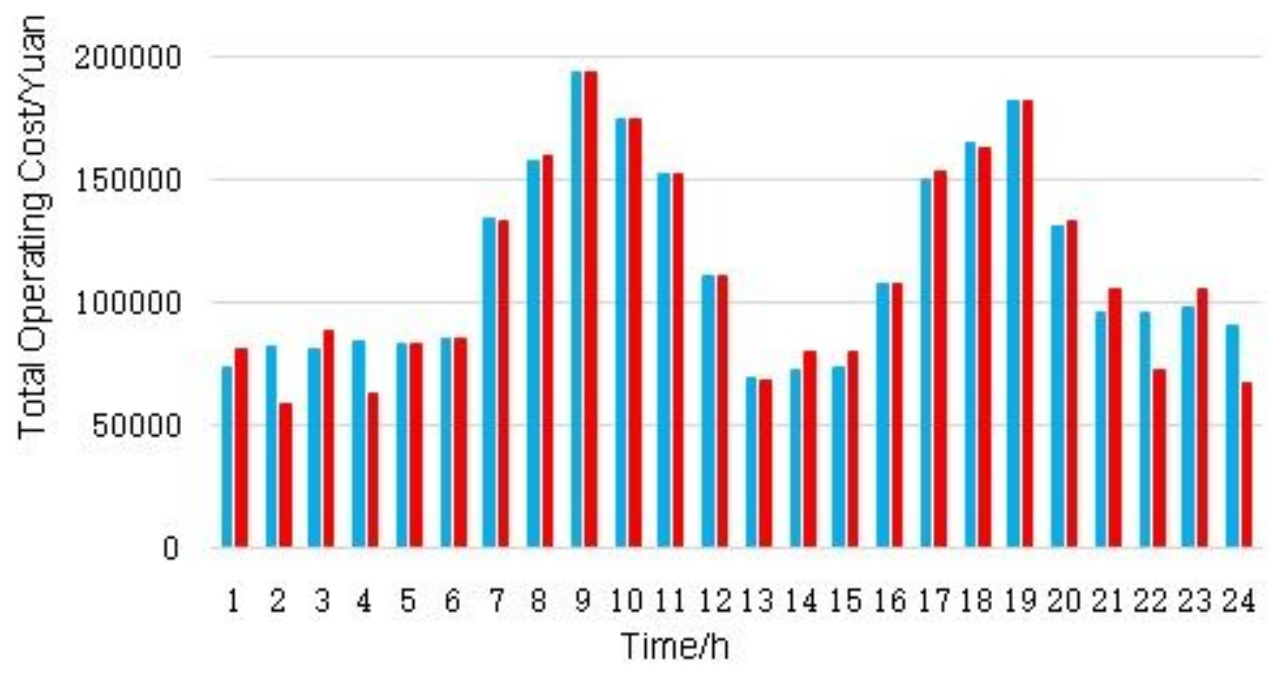

- Scenario 1 Total Operating Cost $=$ Scenario 2 Total Operating Cost

\section{Figure 14}

System Heat Output and Total Operation Cost for Two Scenarios

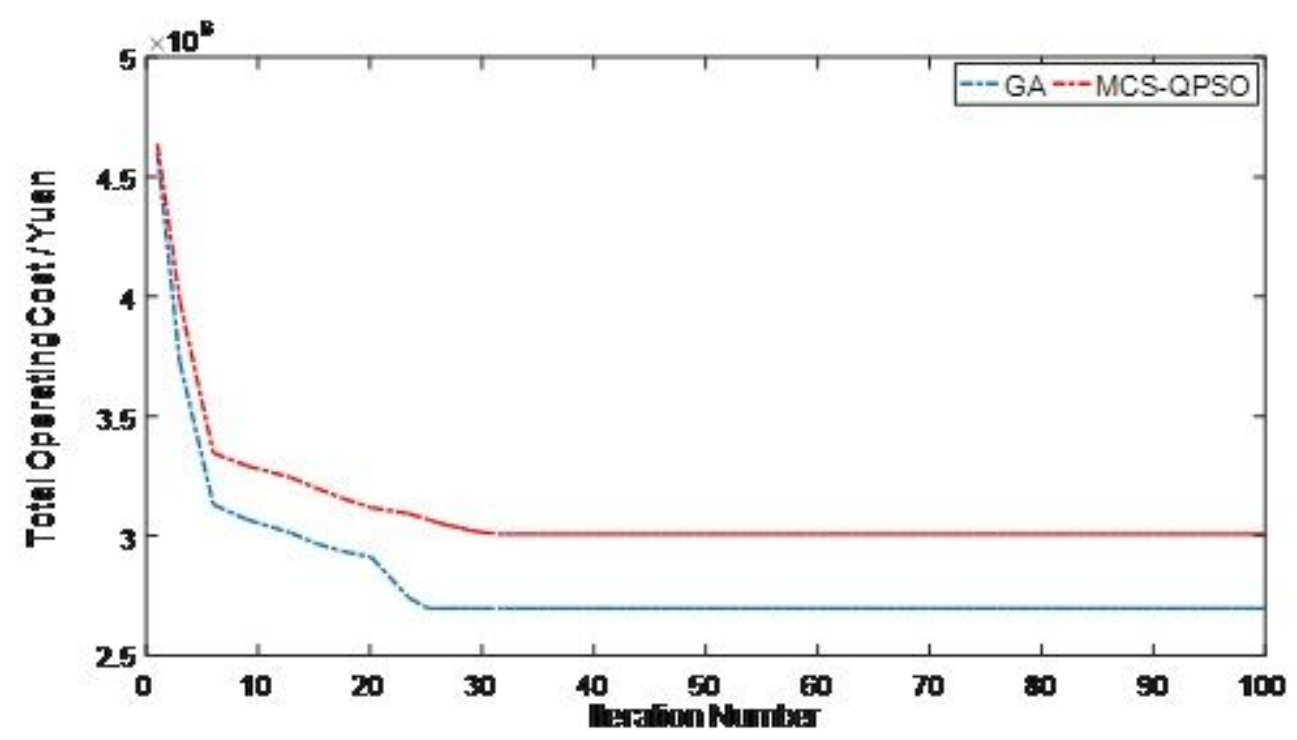

Figure 15

Comparison of iterative curves for different algorithms

\section{Supplementary Files}

This is a list of supplementary files associated with this preprint. Click to download.

- Replytoeditorialcomments.docx 